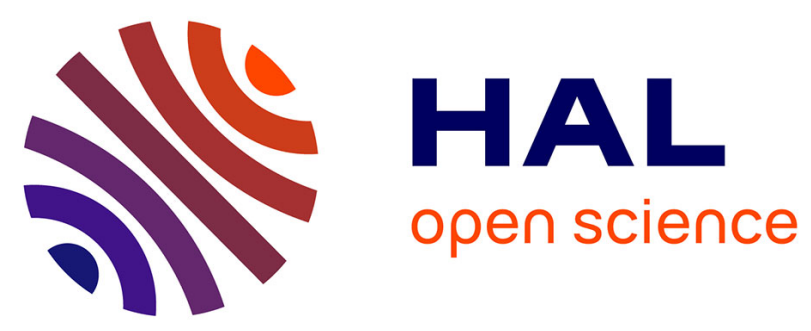

\title{
An electrostatic vertical microscanner for phase modulating array-type Mirau microinterferometry
}

Justine Lullin, Sylwester Bargiel, Patrice Moal, Stéphane Perrin, Jorge Silvestre, Nicolas Passilly, Luc Froehly, Franck Vieudrin, Christophe Gorecki

\section{- To cite this version:}

Justine Lullin, Sylwester Bargiel, Patrice Moal, Stéphane Perrin, Jorge Silvestre, et al.. An electrostatic vertical microscanner for phase modulating array-type Mirau microinterferometry. Journal of Micromechanics and Microengineering, 2015, 25 (11), pp.115013. 10.1088/0960-1317/25/11/115013 . hal-02131371

\section{HAL Id: hal-02131371 \\ https://hal.science/hal-02131371}

Submitted on 16 May 2019

HAL is a multi-disciplinary open access archive for the deposit and dissemination of scientific research documents, whether they are published or not. The documents may come from teaching and research institutions in France or abroad, or from public or private research centers.
L'archive ouverte pluridisciplinaire HAL, est destinée au dépôt et à la diffusion de documents scientifiques de niveau recherche, publiés ou non, émanant des établissements d'enseignement et de recherche français ou étrangers, des laboratoires publics ou privés. 


\title{
Electrostatic vertical microscanner for phase modulating array-type Mirau microinterferometry
}

\author{
Justine Lullin ${ }^{1, *}$, Sylwester Bargiel ${ }^{1}$, Patrice Lemoal ${ }^{1}$, Stéphane Perrin ${ }^{1}$, Jorge Albero ${ }^{1}$, Nicolas \\ Passilly $^{1}$, Luc Froehly ${ }^{1}$, Franck Lardet-Vieudrin ${ }^{1}$ and Christophe Gorecki ${ }^{1}$ \\ ${ }^{1}$ FEMTO-ST Institute (UMR CNRS 6174, UBFC), 15B Avenue des Montboucons, 25030 Besançon, \\ France \\ *justine.lullin@femto-st.fr
}

\begin{abstract}
We present a micromachined vertical scanner with a $4 \mathrm{x} 4$ array of suspended "spider-type" micromirrors which is a key component of a multi-channel "active" Mirau microinterferometer. The microscanner provides for the vertical displacement of the reference micromirrors for the implementation of phase shifting technique. Vertical comb-drive structures are employed for actuation of micromirrors (displacement range of 1 hundreds of $\mathrm{nm}$ at $500 \mathrm{~Hz}$ ) as well as for in situ capacitive displacement sensing. Integration of micro-opto-electro-mechanical microscanners in handheld interference microscope has the advantage to significantly decrease instrument size because of the small footprint of the microscanner. Particularly interesting applications are low-cost and portable microsystems for parallel inspection of biological tissues, e.g. early diagnosis of specific diseases such as skin cancers.
\end{abstract}

\section{Introduction}

The interferometric techniques are fundamental to the optical metrology due to their unique capability of non-destructive and high-accuracy measurement of a broad range of quantities. An increasing demand for the reduction of system size (portability), lower cost as well as several new metrology challenges, such as in-vivo imaging, have made the miniaturization of interferometric systems an important issue. The combination of the array-type microinterferometers and interferometry-based imaging methods enables construction of portable systems for in-vivo medical diagnostics. The swept source optical coherence tomography (SS-OCT) is a particularly interesting candidate since it allows a fast and non-invasive 3 -D reconstruction of tissue morphology within significant penetration depth $(\sim 1 \mathrm{~mm})$ and high resolution $(5-10 \mu \mathrm{m})$ [1]. Moreover, the implementation of the phase shifting interferometry (PSI) technique [2] with "active" microinterferometer allows to improve sensitivity and to remove some drawbacks of the SS-OCT technique, such as the presence of artefacts in tomography images. PSI techniques are particularly efficient to remove disturbing interferometric terms such as the mirror term, the DC term and the auto-correlation term. Then, this technique leads to an increase of the signal dynamic.

For this purpose, the micro-opto-electro-mechanical (MOEMS) approach offers not only size and cost reduction, but also the implementation of actuated micromirrors, enabling spatial scanning of the light beam in MEMS-based endoscopic probes [3] as well as precise generation of the optical phase shift. In the literature, most phase-shifters for interferometric applications were proposed based on electrostatic comb-drive actuators [4-7]. In 2003, Lee et al. were the first to propose a phase-shifter for interferometric systems based on the levitation force produced by electrostatic comb-drive with in situ parallel plate capacitive sensing [4]. 
In 2006, Choo et al. proposed a phase-shifter based on vertical electrostatic comb-drive made out of silicon. This technology presents a great advantage of simplicity [5]. Finally, Chiou et al. presented a 3x3 array of individually actuated surface-micromachined micromirrors based on the same actuation technology than in [5] for holographic data storage. The absence of position sensing in the two latter papers is a problem for the implementation of PSI algorithm. For this purpose, Oda et al. demonstrated in 2012 a phase-shifter with in situ position sensing based on silicon vertical comb technology. However, these phase-shifters are presented as one optical component but no integration strategy in the final microinterferometer is presented. In particular, MOEMS and MEMS components can be stacked using a multi-wafer vertical integration method to build the array-type MOEMS-based microinterferometers. The potential of such integration has been demonstrated recently for an electrostatically tuned grating microinterferometer for nm-scale displacement measurements [8] as well as a miniature laser Twyman-Green interferometer [9] and 5x5 array of low-coherence Mirau microinterferometers [10]. The last two devices have been developed for a massively parallel in-line inspection of MEMS. As a global strategy of integration of a microinterferometer, the Mirau configuration is well suited due to its vertical architecture and integration capability. However, this transmission-based configuration also imposes specific constraints such as a need of clear optical area around the micromirrors. Recently, the design of an "active" single channel Mirau interferometer has been proposed by Xu et al [11]. However, the fabrication and the characterisation were not presented. Finally, the proposed design did not integrate the objective lens.

In this paper, we present a Silicon-On-Insulator (SOI)-based electrostatic vertical microscanner that generates a vertical displacement of a large platform with a $4 \mathrm{x} 4$ array of reference micromirrors for the PSI-based imaging in SS-OCT system with in situ position sensing. In the following sections, we describe in details the technology of vertical comb-drive structures [12], that are employed for both actuation and sensing purposes as well as the results of static and dynamic characterization.

\section{Design and simulation}

\section{A. Integration of the microscanner in the microinteferometer}

The vertical microscanner is a part of a global optical system and must meet its specifications. The design of the optical coherence tomography system is defined in details in [13]. Figure 1 presents schematically the construction of a multi-channel $4 \times 4$ Mirau microinterferometer, in which the microscanner is implemented. The proposed microinterferometer is based on the vertical multi-wafer integration technology and consists of a doublet of plano-convex glass lenses, a microscanner wafer with array of reference micromirrors, spacer and planar beam splitter. The light is coming from the top, focused by the lens partly in the skin and partly on the reference micromirror thanks to the beam splitter. The light going back from the skin and from the mirror recombine at the beam splitter and interfere. The sum of the two light beams is recovered by the Mirau lens. The interferometric signal, recorded by a camera, is used to reconstruct a 3D image of the sample. Then the array of 16 micromirrors gives 16 3D images of the sample. The lateral dimensions of each 3D image are defined by the micromirror size and its vertical dimension is linked to the swept-source parameters. In-plane stitching is realized in order to scan a whole 3D volume of skin. In order to use phase shifting technique, the microscanner generates the vertical movement of the whole $4 \mathrm{x} 4$ array of reference micromirrors at a specific frequency. The applied phase modulating algorithm was proposed by $\mathrm{P}$. Bu and al. in [14]. This method is less sensitive to phase shift errors than standard phase shifting algorithm since it is based on the amplitude and frequency modulation of the light created by the sinusoidal actuation of the reference mirror. The algorithm is based on the acquisition of four interference images. Moreover, unlike classical four steps phase shifting algorithm that requires a linear motion of the phase-shifter [5], this algorithm is adapted to sinusoidal motion. The sequence of images is taken by a camera during one half of an 
actuation period, then the actuation frequency must be at least 8 times slower than the camera. Here, the frame rate of the camera is set to $4 \mathrm{kfps}$ and the actuation frequency to $500 \mathrm{~Hz}$. In order to benefit from the high quality factor of the resonant frequency, the system is designed to work at resonance. Finally, the micromirror peak-to-peak displacement amplitude is set to $2 \times \frac{2.63 \lambda}{4 \pi}$, which is the optimum value for the implementation of this algorithm as defined in [15]. The $\lambda_{0}$ and $\delta \lambda$ of the swept light source is $840 \mathrm{~nm}$ and $50 \mathrm{~nm}$, respectively. Thus, the amplitude of the micromirror displacement is $352 \mathrm{~nm}$.

The construction of the microscanner has to fulfil several requirements related to the vertical integration within the Mirau microinterferometer. First, the microscanner wafer must be positioned up-side down in the wafer stack to ensure that the reference mirror faces the beam splitter (Fig. 1). The specificity of this microscanner design is that it works in transmission, i.e. the area around the micromirror should be transparent whereas microscanners presented in the literature usually work in reflection. Moreover, microscanner wafer must be compatible with the overall integration strategy based on anodic bonding (materials, process temperature, surface quality)(Fig. 2). Finally, electrical interconnections is provided to drive the actuators and sensors by external electronics. For this purpose, holes in the spacer are placed in such a way to enable side access to the contact pads, located on the border of each chip, during wire bonding [16].

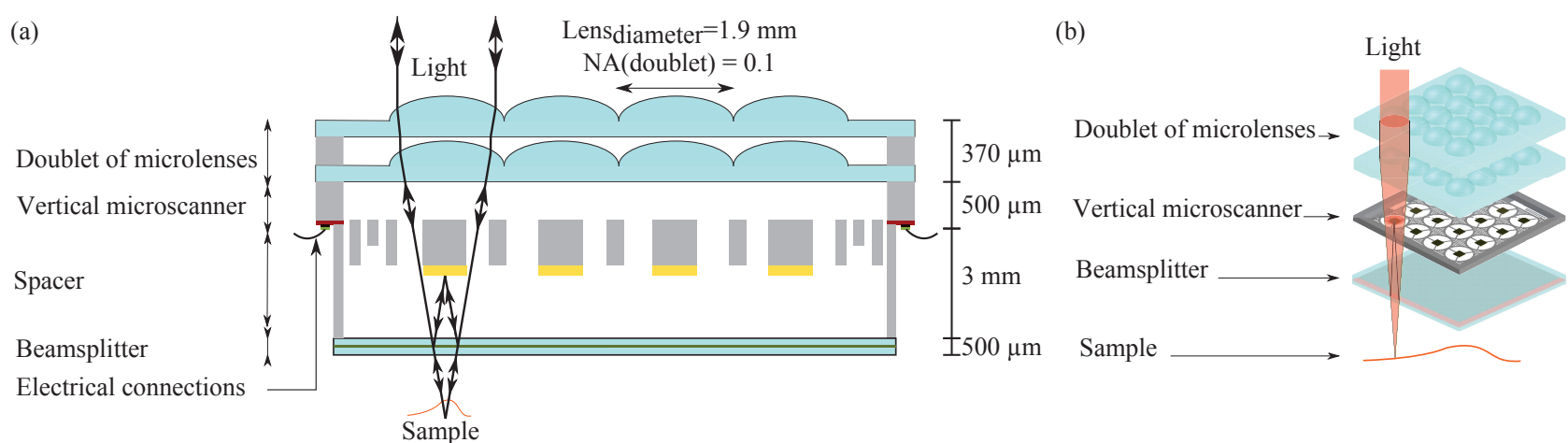

Fig. 1. Integration of the vertical microscanner within multi-channel "active" Mirau microinterferometer: (a) schematic cross-sectional view, (b) 3D expanded view.

(a)

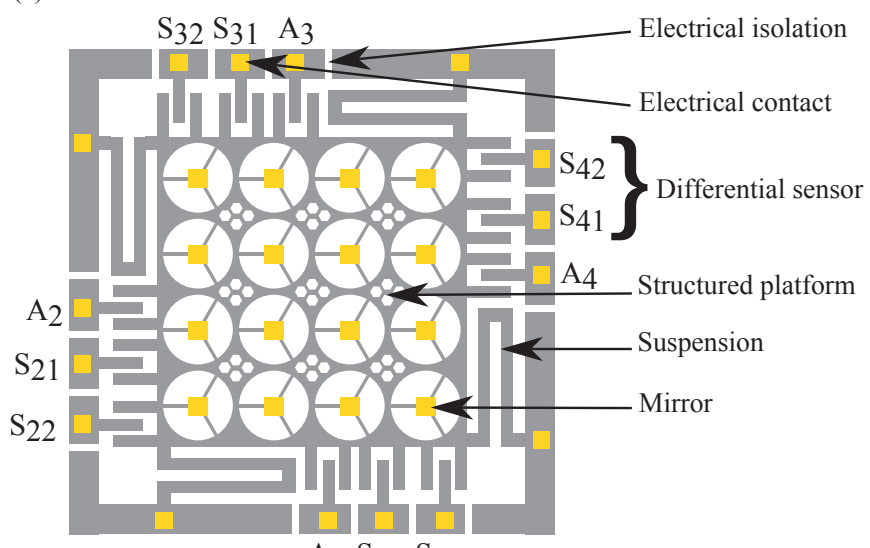

(b)

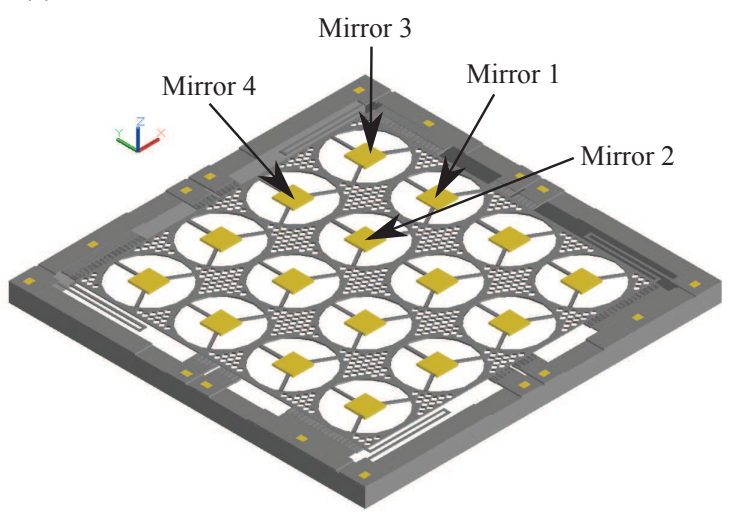

Fig. 2. Electrostatic vertical microscanner with $4 \times 4$ array of reference micromirrors: (a) Schematic top view (not in scale), (b) corresponding 3D view (in scale). 


\section{B. The micromirror platform}

The size and the shape of the micromirrors are defined by the optical design of the Mirau microinterferometer as a $400 \mu \mathrm{m} \times 400 \mu \mathrm{m}$ square. However, in this specific work, in-plane stitching is realized in order to reconstruct a 3D image with total lateral dimensions equal to $8 \mathrm{~mm} \times 8 \mathrm{~mm}$. Therefore, square micromirrors are preferred in order to facilitate digital processing of acquired X/Y images. Important design issue concerns the minimization of the obscuration created by the micromirror in the center of the light beam. Moreover, the light beam is focused in the skin by the doublet of $2 \mathrm{~mm}$ diameter glass lenses, with a numerical aperture of the doublet of 0.1 [17]. The size of the square micromirror corresponds to a transmission of the mirror plane of $94 \%$ (the clear aperture around the micromirror having a diameter of $1.84 \mathrm{~mm}$ ), which is a typical value when compared to reference literatures $(70 \%-80 \%)[18,19]$. As explained before, the implementation of PSI method implies that the micromirror cannot be simply deposited on the backside of the lenses [10] but must be integrated onto an actuated platform and a clear aperture around the micromirror is needed. Two solutions are then possible, either the micromirrors are deposited on a transparent membrane [20] or they are suspended by suspensions. Integration of a glass membrane into an actuated silicon structure is a technological challenge and complicates substantially the fabrication. Therefore, the a suspended mirror is chosen. The suspensions, referred to as spider legs, obscure the light beam and create diffraction. However, it has been shown that their impact is negligible in a certain range of design parameters such as their width, number and radius of curvature [21]. According to the results of [21], the suspensions are chosen to be $50 \mu \mathrm{m}$ wide and have a radius of curvature equal to the clear aperture diameter around the micromirror. Finally, the platform is dimensioned to the array of micromirrors, vertically aligned with the lenses, forming a $8 \mathrm{~mm} \times 8 \mathrm{~mm}$ square. The platform is formed in a $40 \mu \mathrm{m}$ thick device layer of the SOI wafer and is structured with holes in order to decrease its weight. Indeed the resonant frequency of the actuated platform with micromirrors is related to the mass and the suspensions stiffness according to the following equation:

$f_{\text {resonant }}=\frac{1}{2 \pi} \times \sqrt{\frac{k}{m}}$. If $m$, the mass, is reduced, then $k$ is reduced and the vertical actuation force equal to $F_{z}=k_{z} \times z$ is minimized and so the actuation energy. A honeycomb structure is chosen for its good trade-off between mass and stiffness, preventing the possible vertical deformation of the platform. The static deformation of the mirror platform has been studied in static (Fig. 3). The maximum vertical deformation equals $140 \mathrm{~nm}$ which is much smaller than the axial resolution of the system that is $6 \mu \mathrm{m}$. Then this static deformation has no impact for the imaging system. Moreover optical simulations, made with Zemax, were done taking into account a tilt tolerance of 0.05 degree [13]. The angle, created by the vertical displacement simulated here, corresponds to a maximum micromirror angle of 0.002 degree, which is below the optical tolerance specification. 


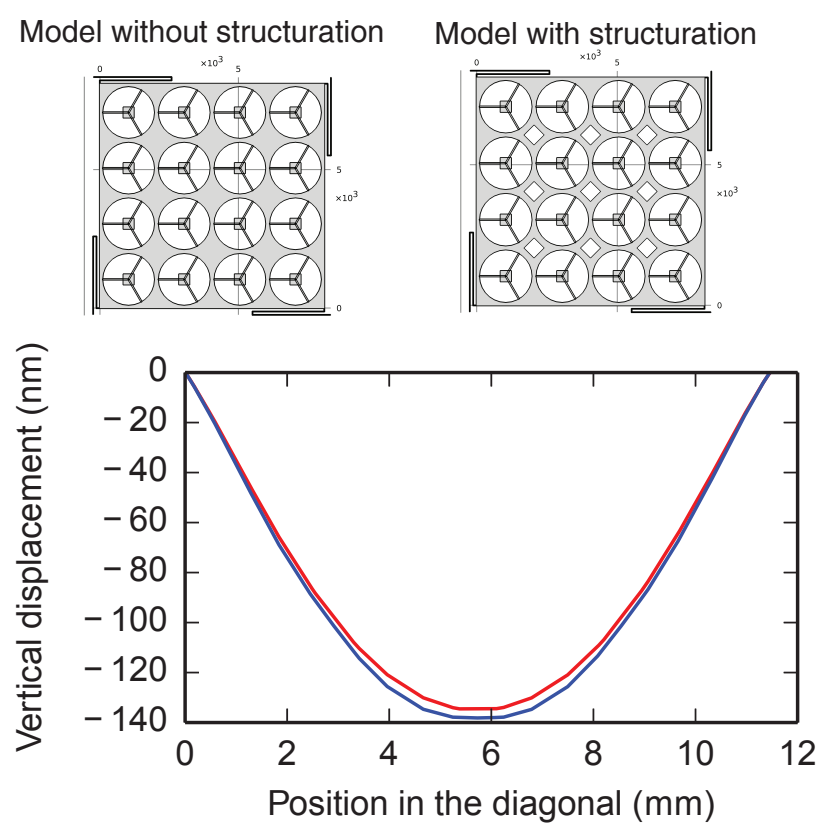

Fig. 3. Impact of structuration of the suspended platform on its vertical static deformation (diagonal direction). Two simulations are performed to analyse two limit cases in terms of structuration: one without any structuration of the platform and one with nine $700 \mu m \times 700 \mu m$ square holes centered between the optical apertures. These two simulations represent two limit cases in term of structuration.

\section{The suspensions}

In order to avoid in-plane parasitic movement of actuated micromirrors, spring suspensions have been designed to be stiff in the in-plane directions. Additionally, asymmetric multi-folder springs are employed due to their small foot print (Fig. 4). The vertical stiffness is set by the targeted resonant frequency $(500 \mathrm{~Hz} \pm 50 \mathrm{~Hz})$ and the mass of the platform. The springs are placed around the platform so that the in-plane total stiffness is the same in $\mathrm{x}$ and $\mathrm{y}$ directions. In the following, silicon is modelled as an isotropic material which Young Modulus is equal to $170 \mathrm{GPa}$ and Poisson's ratio 0.26. However it has been checked that simulation results with an anisotropic and an isotropic hypothesis are very close.

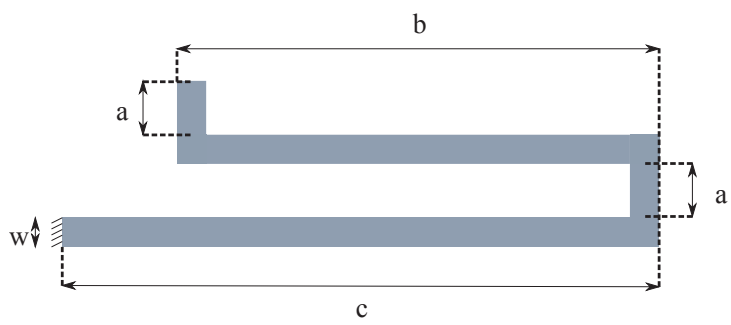

Fig. 4. Asymmetric springs dimensions.

\begin{tabular}{c|l|c} 
Symbol & Description & Value \\
\hline $\mathrm{w}$ & Beam width & $30 \mu \mathrm{m}$ \\
$\mathrm{t}$ & Beam thickness & $40 \mu \mathrm{m}$ \\
$l_{1}=a$ & Beam 1 length & $90 \mu \mathrm{m}$ \\
$l_{2}=b$ & Beam 2 length & $2610 \mu \mathrm{m}$ \\
$l_{3}=a$ & Beam 3 length & $90 \mu \mathrm{m}$ \\
$l_{4}=c$ & Beam 4 length & $2820 \mu \mathrm{m}$
\end{tabular}

Table 1. Design parameter of the asymmetric springs.

Simulations using Comsol and theoretical calculations have been performed in order to design the spring according to the specifications (Table 1). Theoretical values have been retrieved using the energy method. This method is well detailed in [22] and is based on Castigliano's second theorem. To use this method, the serpentine spring is decomposed in 4 straight beams that are analysed. The total strain energy of the linearly elastic structure is calculated as follows: 


$$
U=\frac{1}{2}\left(\sum_{i=1}^{n} \int_{0}^{l_{i}} \frac{M_{i}^{2}}{E I_{i}} d \epsilon+\sum_{i=1}^{n} \int_{0}^{l_{i}} \frac{T_{i}^{2}}{G J_{i}} d \epsilon\right)
$$

where $l_{i}$ is the length of beam $m_{i}, M_{i}$ is the Moment of beam ${ }_{i}, T_{i}$ is the Torsion of beam $i, I_{i}$ is the Moment of Inertia of beam ${ }_{i}, J_{i}$ is the Torsion constant of beam $i$ and $\mathrm{G}$ and $\mathrm{E}$ are respectively the shear modulus and the Young modulus of the material.

For a rectangle cross-section, $I=\frac{w t^{3}}{12}$ and $J=\frac{1}{3} w^{3} t\left(1-\frac{192 w}{\pi^{5} t} \sum_{i=1, i \text { odd }}^{\infty} \frac{1}{i^{5}} \tanh \left(\frac{i \pi t}{2 w}\right)\right)$.

The moment and the torsion of each beam, created by $M_{0}, T_{0}$ and $F_{z}$ are defined as follows:

$$
\begin{aligned}
& M_{1}=M_{0}-F_{z} \epsilon \quad T_{1}=T_{0} \\
& M_{2}=T_{0}+F z \epsilon \quad T_{2}=M_{0}-F_{z} l_{1} \\
& M_{3}=M_{0}-F_{z}\left(\epsilon+l_{1}\right) \quad T_{3}=F_{z} l_{2}+T_{0} \\
& M_{4}=F_{z} *\left(l_{2}-\epsilon\right)+T_{0} \quad(2) \quad T_{4}=M_{0}-l_{1} F_{z}-l_{3} F_{z}
\end{aligned}
$$

With the conditions:

$$
\frac{\partial U}{\partial M_{0}}=0, \frac{\partial U}{\partial T_{0}}=0 \text { and } \frac{\partial U}{\partial F_{z}}=\delta z
$$

and $l_{1}=l_{3}=a, l_{2}=b$ and $l_{4}=c$, one obtains,

$$
\begin{gathered}
k_{z}=\frac{F_{z}}{\delta z}=\frac{12 E G I J(2 E I a+G J(b+c))(E I(b+c)+2 G J a)}{S U M} \\
S U M=12 E^{3} I^{3} a^{2} b\left(2 a c+b^{2}+b c\right) \\
+4 E^{2} G I^{2} J a\left(4 a^{3} b+16 a^{3} c+6 a^{2} b^{2}+3 a b^{2} c+3 a b c^{2}+2 b^{4}+5 b^{3} c-b c^{3}+2 c^{4}\right) \\
+E G^{2} I J^{2} \quad\left(32 a^{5}+8 a^{3} b^{2}+40 a^{3} b c+32 a^{3} c^{2}+16 a^{2} b^{3}+24 a^{2} b^{2} c-24 a^{2} b c^{2}\right. \\
\left.+16 a^{2} c^{3}+b^{5}+5 b^{4} c-2 b^{3} c^{2}-2 b^{2} c^{3}+5 b c^{4}+c^{5}\right) \\
\left.+2 G^{3} J^{3} a \quad\left(8 a^{3} b+8 a^{3} c+b^{4}+4 b^{3} c-6 b^{2} c^{2}+4 b c^{3}+c^{4}\right)\right)
\end{gathered}
$$

Following the same method, we calculate $k_{x}$ and $k_{y}$. Table 2 summarizes the obtained results.

The mode shapes of the actuated platform are simulated to confirm stiffness calculations. The first mode is confirmed to be the piston mode at $527 \mathrm{~Hz}$, whereas the second mode and third mode are torsion modes at $840 \mathrm{~Hz}$ (Fig. 5). 
Table 2. Calculated and simulated stiffness of the suspension. The calculated mass $m$ of the suspended platform is equal to $2 \cdot 46 \cdot 10^{-6} \mathrm{~kg}$ which corresponds to a frequency of $530 \mathrm{~Hz}(533 \mathrm{~Hz})$.

Mode 1

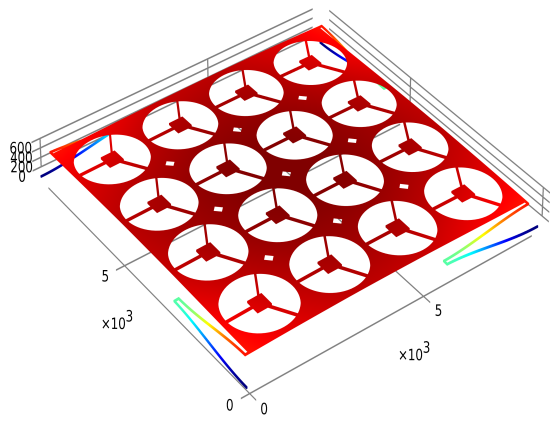

Mode 2

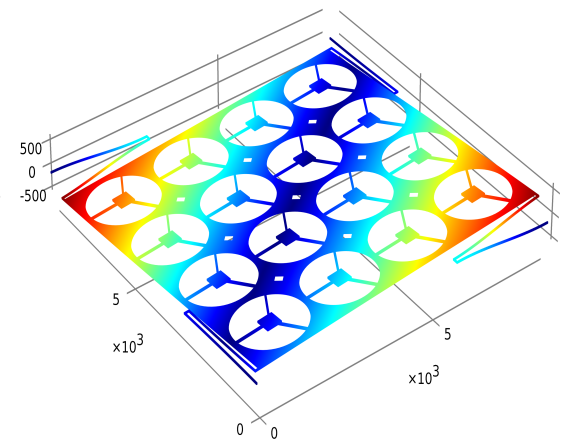

Mode 3

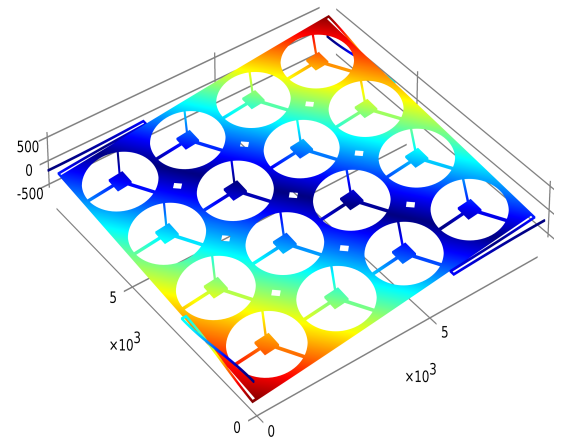

Fig. 5. Simulation of the three first natural modes of the vertical microscanner.

\section{The actuator and sensor}

Actuating and sensing are the core functions of the device. In order to use the phase shifting algorithm, it is necessary to know the displacement amplitude of the micromirrors. Therefore, a capacitive sensor is also integrated to the structure. Both the vertical actuators and sensors are based on the electrostatic field created by vertical comb fingers (Fig. 6). This technology has been intensively used due to its relative easiness of implementation in silicon-based MEMS [7,23,24]. Indeed, combination of SOI substrates and Deep Reactive Ion Etching (DRIE) allows fabrication of both the micromechanical structures as well as the electrostatic actuators and sensors.

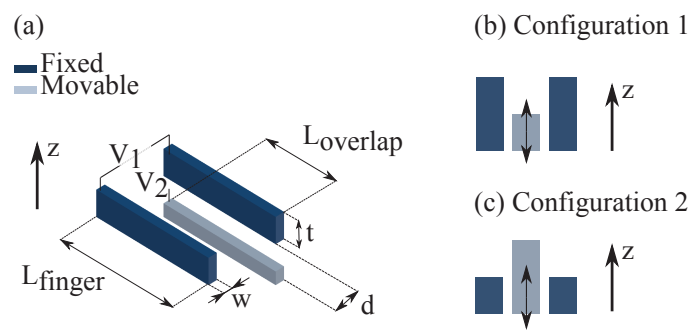

Fig. 6. Assymetrical comb finger design : (a) Dimensions design, (b) Configuration 1, c) Configuration 2. 
Actuator The vertical electrostatic force is created by a change of capacitance in the vertical direction. This is possible when a vertical asymmetry exists in the design of the comb fingers (Fig. 6, (b)). If parallel plate capacitances are used to create lateral electrostatic actuator, here the fringing capacitances are responsible for the actuation. The electrostatic force, between two opposite fingers, is proportional to the derivative of the capacitance between these fingers according to the following relationship:

$$
F_{e l}=\frac{1}{2} \frac{\partial C}{\partial z} V^{2}
$$

where $C$ is the capacitance and $\mathrm{V}$ is the potential between the two fingers.

Sensor The sensing function is created by differential capacitance. It has been shown that designing two specific types of comb fingers (Fig. 6), their differential capacitance is linear as a function of the only vertical displacement of the comb fingers [23]. When the movable finger moves up, the capacitance mainly changes for the Configuration 2 (Fig. 6, c)) whereas if the movable finger moves down then the capacitance mainly changes for the configuration 1 (Fig. 6, (b)). This method is used to design the four differential sensors, each composed of one set of fingers with the Configuration 1 and another with Configuration 2, that are placed around the movable platform as shown in Fig. 2.

Simulations Simulations of the capacitance have been performed with 3D FEM simulation using Intellisuite to study both the actuation and the sensing functions since they are based on the same physical phenomena. A model composed of one movable finger facing two fixed fingers were design. The ThermoElectro-Mechanical module was used. A MacroModelExtraction/Capacitance vs. displacement analysis and a Static/Electro Thermo Relaxation analysis were chosen to process the capacitance change and the static displacement of the platform as a function of an applied voltage between fixed and moving parts. Then Figure 7 shows the capacitance change for each of comb finger configurations with single movable finger versus the platform displacement as well as their differential capacitance.

On the one hand, linear fitting of the differential curve allows to determine the sensitivity of one couple of fingers $0.64 \mathrm{fF} / \mu \mathrm{m}$ and then calculate the sensitivity of a differential sensor, made of 34 couples of fingers, to be $22 \mathrm{fF} / \mu \mathrm{m}$. The same simulation has been performed for a gap equal to $4 \mu \mathrm{m}$ and a sensitivity of 0.81 $\mathrm{fF} / \mu \mathrm{m}$, corresponding to a total sensitivity of $27 \mathrm{fF} / \mu \mathrm{m}$ for one differential sensor, has been obtained. It is worth to note that the differential capacitance in the range of some $\mathrm{fF}$ has to be measured for required by PSI displacement amplitude.

On the other hand, the static displacement of the platform as a function of the applied voltage was simulated, based on a capacitance change simulation, for two finger gaps (4 and $5 \mu \mathrm{m})$, and compared to the experimentally obtained values (Fig.19).

The results of the capacitive simulation are coherent with the literature results (Sensitivity $=0.66 \mathrm{fF} / \mu \mathrm{m}$ and $0.68 \mathrm{fF} / \mu \mathrm{m}$ respectively in [24] and in [7] for similar parameters and technology) and are used to chose the design parameters of the comb fingers (Table 3 ). 


\begin{tabular}{c|l|c} 
Symbol & Description & Value \\
\hline$L_{\text {finger }}$ & Finger length & $200 \mu \mathrm{m}$ \\
$L_{\text {overlap }}$ & Finger overlap & $190 \mu \mathrm{m}$ \\
$\mathrm{d}$ & Gap & $5 \mu \mathrm{m}$ \\
$t$ & Finger thickness & $40 \mu \mathrm{m}$ \\
$w$ & Finger width & $10 \mu \mathrm{m}$ \\
$N_{\text {actuator }}$ & Total movable finger number & 400 \\
$N_{\text {sensor }}$ & Total movable finger number per sensor type & 136
\end{tabular}

Table 3. Design parameter of the asymmetric comb finger

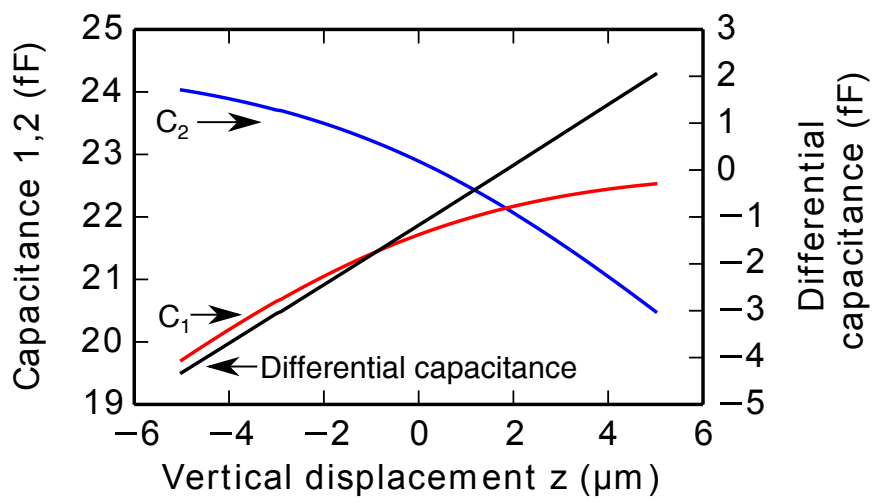

Fig. 7. Capacitance versus displacement of Configuration 1 and Configuration 2 and differential capacitance for one movable finger facing two fixed fingers. Linear fitting of the differential capacitance gives a sensitivity of $0.64 \mathrm{fF} / \mu \mathrm{m}$.

\section{Fabrication of the device}

The technology of vertical scanner is based on double-side processing of SOI wafer using DRIE method. The SOI substrate has been chosen since it ensures well-controlled thickness of device layer $(40 \pm 0.5 \mu \mathrm{m})$, in which the designed micromechanical structures will be formed. Indeed, the thickness parameter is of great importance for vertically actuated structures because of the spring stiffness sensitivity and the necessity to control a vertical offset between the comb fingers.

The first stage of the scanner fabrication, i.e. structuration of device layer, depends directly on the fabrication method of asymmetric comb fingers. In this work, it is realized by a two-steps DRIE process (Fig. 8). First, two mask layers, one in a silicon dioxide $(\mathrm{t}=0.8 \mu \mathrm{m})$ and the other in a photoresist $(\mathrm{t}$ $=2.5 \mu \mathrm{m}$ ), are formed on the device layer in two subsequent photolithography procedures (Fig. 8, steps (a)). The alignment precision achieved was $\pm 0.75 \mu \mathrm{m}$. These mask patterns, corresponding to the two different sets of the facing fingers, are used in the first DRIE process to etch all comb fingers down to the Buried Oxide (BOX) layer $\left(t_{d l}=40 \mu \mathrm{m}\right)$. Simultaneously, other micromechanical structures, i.e. the springs, the platform and the mirrors, are also realized. After stripping the resist mask, one set of comb fingers is still protected by $\mathrm{SiO}_{2}$ mask, whereas the other is exposed to the etching plasma during the second DRIE process. In consequence, the thickness of unprotected fingers is reduced by half $\left(\frac{1}{2} t_{d l}=20\right.$ $\mu m)$, which creates a vertical asymmetry between the facing comb fingers. This second DRIE process, realized with an ICP DRIE machine (Pegasus Rapier SPTS), has been optimized to prevent the notching effect due to the BOX. The actual gap between finger was measured to be $4 \mu \mathrm{m}$, instead of the targeted $5 \mu \mathrm{m}$, because of an overestimated under-etching during masks creation and silicon etching. Finally, an 
(a)

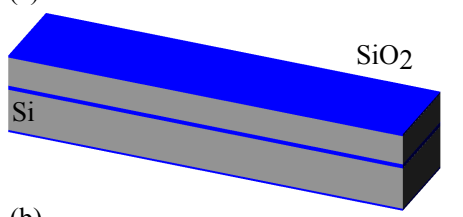

(b)

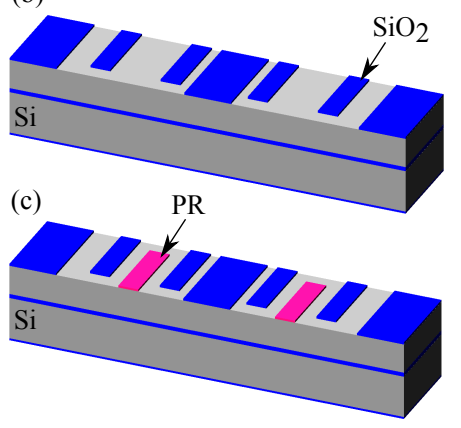

(d)

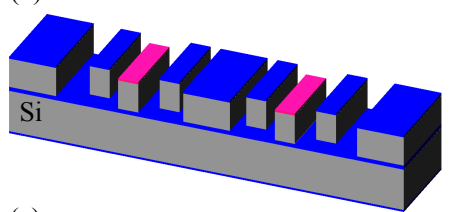

(e)

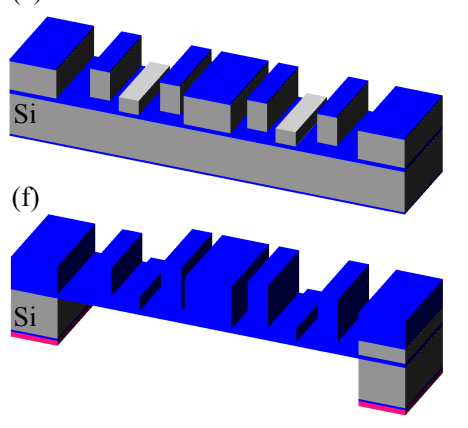

(g)

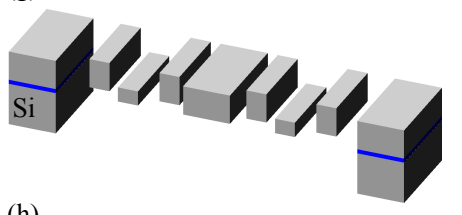

(h)

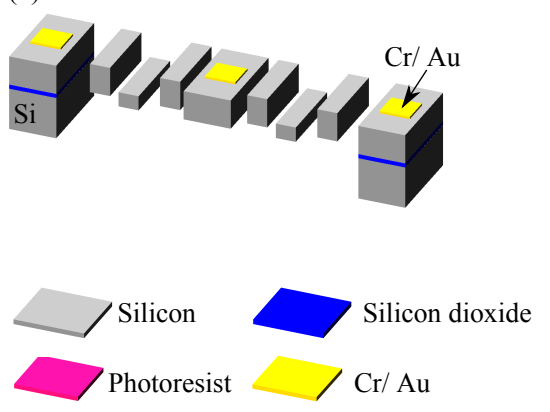

Fig. 8. Flow chart of the fabrication process: (a) oxydation of SOI wafer $(800 \mathrm{~nm})$, (b) Photolithography and ICP DRIE for structuration of $\mathrm{SiO}_{2}$ layer (Mask 1), c) Photolithography of S1828 photoresist layer (Mask 2), d) First DRIE, e) Removal of Mask 2 and Second DRIE, f) Oxidation of the wafer (200 nm) to protect front side during back side release followed by back side DRIE with thick photoresist mask, g) Vapor HF etching of $\mathrm{SiO}_{2}$ layers and BOX, h) Deposition of $\mathrm{Cr} / \mathrm{Au}(10 \mathrm{~nm} / 150 \mathrm{~nm})$ by sputtering though physical mask.

important drawback of this step is linked to its "maskless" character. Realization of classic Bosch process leads in this case to the creation of thin silicon "walls" at the edges of the processed fingers (Fig. 9 B.). This phenomena has already been observed in [23] where a passivation layer was deposited before RIE etching step. One of the possible explanations is that during this second DRIE step, the teflon-like polymer that is deposited on the finger side-walls, has finally no more silicon support and become a mask during the following etching cycle by falling down on the structure. Another explanation would be a micromasking effect, related to high selectivity of silicon etching in SPTS system towards polymer $(S=150)$ and oxide $(S=250)$ masks as well as the deformation of local electric field due to sharp edges of fingers. In [23], solutions were suggested but not tested. Here, the proposed solution relies on increased time of $\mathrm{SF}_{6}$ etching cycle of Bosch procedure in order to systematically under-etch the remaining thin silicon wall (Fig. 9).

The second stage of the scanner fabrication deals with the release of the structure. This part is always very sensitive when it comes to active structure. Here, the back side of the device, i.e. the handle layer $\left(t_{h l}=500\right.$ $\mu m)$, must be completely etched to allow light transmission through the platform, around the micromirrors. This etching step is realized through a $15 \mu \mathrm{m}$ thick AZ9260 resist mask deposited on the $\mathrm{SiO}_{2}$ layer. Note that $\mathrm{SiO}_{2}$ etching step should be preferably done with successive short etching times to limit an elevation of the temperature that leads to difficulties in removing the resist. Moreover, it has been found that some cracks appear in the BOX when it is reached by the etching plasma. The consequence is the penetration of the etching plasma through the BOX layer and then the partial etching of the device layer, destroying the structure (Figure 10). Therefore, a thin thermal silicon dioxide layer $(t=150 \mathrm{~nm})$ is thermally grown to protect the front side during the back-side etching. Finally, the release of the platform is done by etching of the silicon dioxide layers in vapour HF (Fig. 8, step (f)). In order to ensure the best possible quality of micromirror surface as well as contact pads, the deposition of the metal layer is performed as a last step. For the fabrication of micromirrors, the sputtering of $\mathrm{Cr} / \mathrm{Au}$ layer $(10 \mathrm{~nm} / 150 \mathrm{~nm})$ is realized through a physical 
A.

(a)

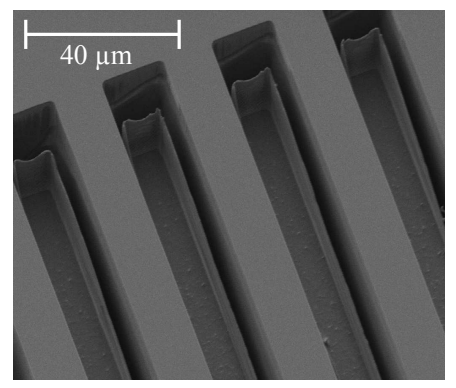

(b)

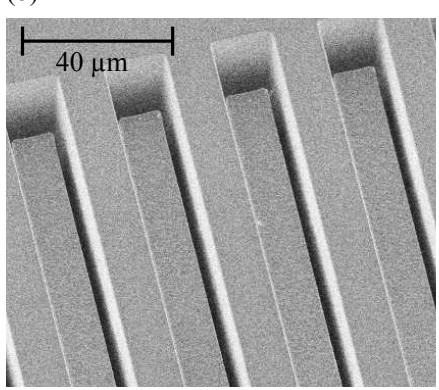

B.

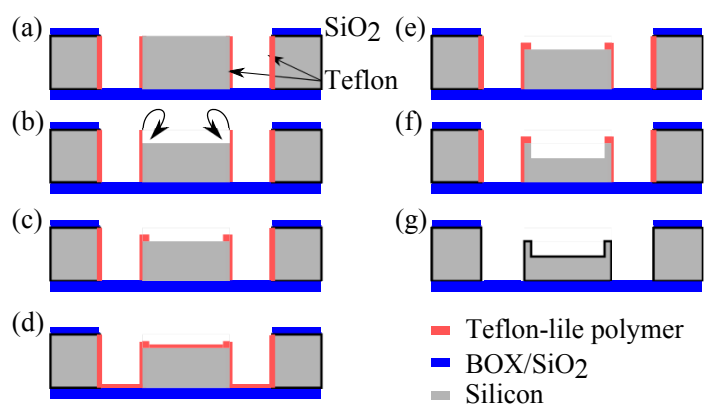

Fig. 9. SEM image of fabricated comb fingers. A) SEM images : (a) Creation of silicon "wall" during second DRIE where there is no masking layer (Fig. 8 step e)) (b) Improved DRIE process to limit silicon "wall" creation. B) Schematic of the formation of silicon walls during DRIE process : (a) Deposition of teflon everywhere and isotropic etching of the teflon on horizontal surfaces, (b) Silicon etching, with formation of free teflon walls, (c) Falling of teflon walls on the silicon, d) Deposition of teflon, (e) Isotropic etching of teflon on horizontal surfaces, some teflon is left, (f) Silicon etching with teflon residue on horizontal surfaces creating silicon walls, (g) Cleaning of the wafer.

mask, aligned thanks to bonding tools (Fig. 11). For this purpose, both the microscanner and physical wafers are aligned directly in a EVG bonding tool using EVG620 mask alignment system, and then brought into contact in EVG501 wafer bonder. The alignment is maintained in the sputtering machine thanks to an adhesive teflon tape. The physical mask has been designed in order to minimize the tolerance sensitivity.

A.

(a)

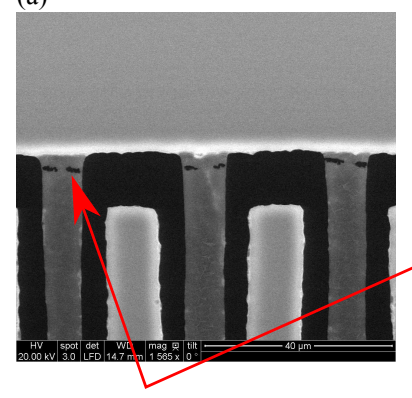

Fingers etched by the back side (b)

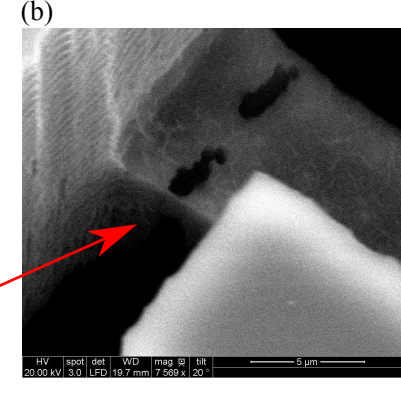

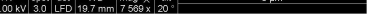

Fig. 10. SEM image after back side DRIE. A : SEM images (a) Comb fingers that are partially etched from the back side, (b) Zoom on one finger. B : Schematic of the etching of comb fingers by the back side through BOX cracks : (a) Comb fingers SOI wafer, (b) Back side DRIE etching down to the BOX, (c) Creation of cracks in the BOX, (d) Penetration of etching plasma through the BOX from the back side to the front side of the wafer resulting in comb fingers destruction.

Two strategies of fabrication were implemented: the first to enable the separation of individual chips from the wafer and thus facilitate the characterization (Fig. 12) and the second for the future wafer-level integration within the Mirau microinterferometer (Fig. 13). With the first design, individual chips were separated using UV tape to maintain them while manually cutting small silicon bridges and sucking up the dust using vacuum system (Fig. 12). Finally, short exposure of tape to UV light allows gentle releasing the chips. One wafer with this "chip" design was fabricated without optimization and a yield of $71 \%$ was obtained. For 
(a)

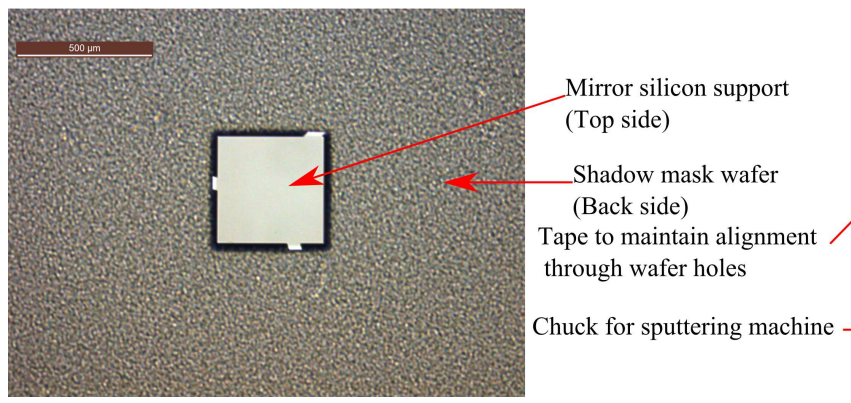

(b)

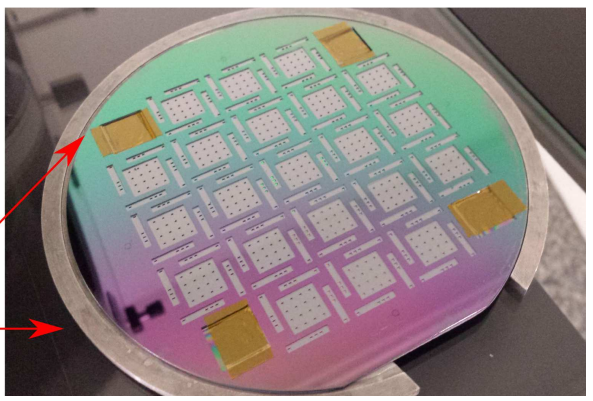

Fig. 11. Deposition of $\mathrm{Cr} / \mathrm{Au}$ layer on micromirrors and pads using shadow mask wafer: (a) image of the shadow wafer aligned with the SOI wafer showing the mirror silicon support, (b) a global view of the aligned wafers (top : shadow mask, bottom : SOI wafer) ready for the mirror sputtering.

the second design, three wafers were fabricated to test the vertical integration of the microscanner with the glass microlenses wafer and the beam splitter. The yield of fabrication of these wafers was $100 \%$ (Fig. 13 (a)).

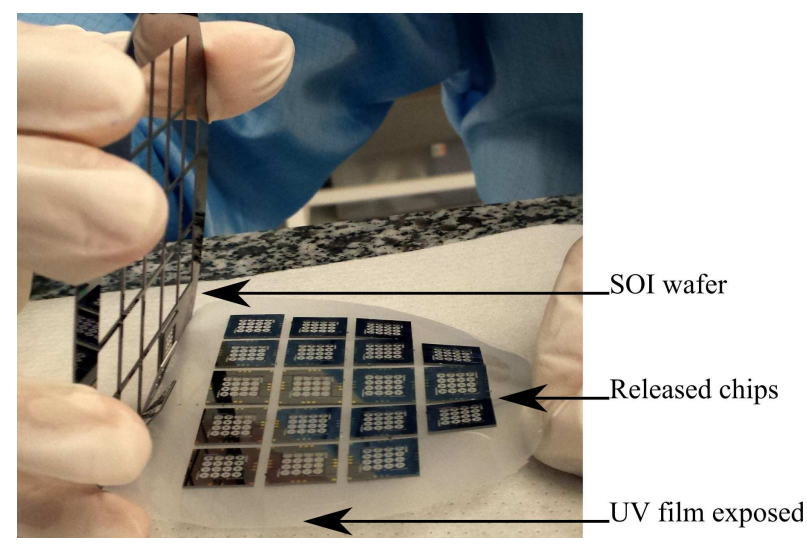

Fig. 12. Design 1: Individual chips separated from the wafer for characterization.

(a)

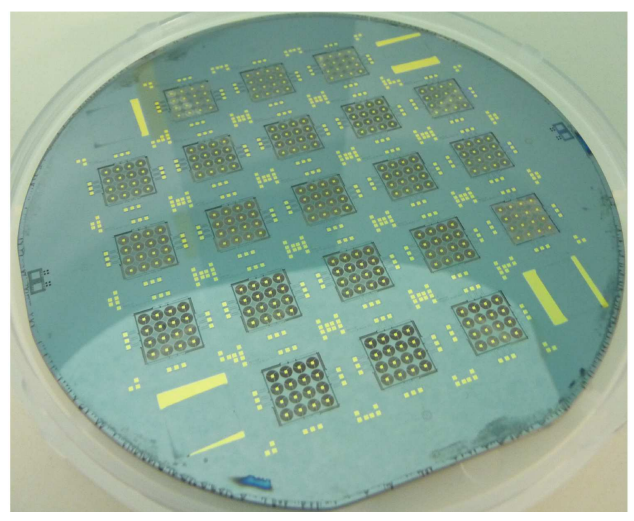

(b)

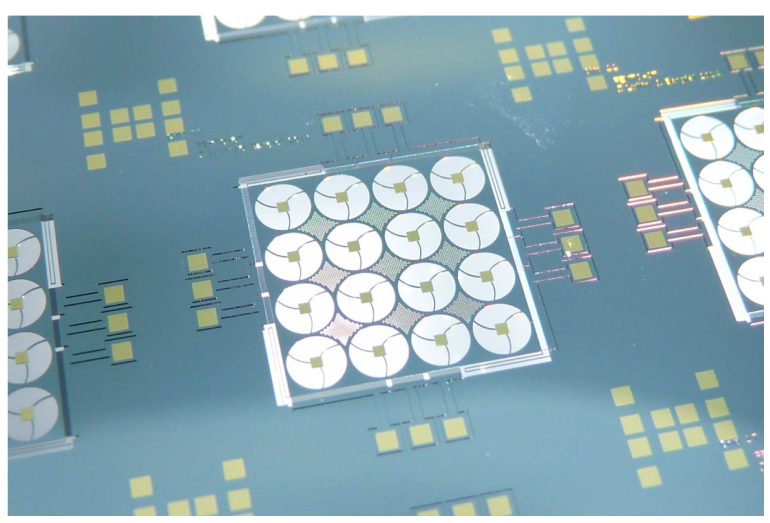

Fig. 13. Design 2: Released microscanner that are kept at wafer level for future vertical wafer-level integration: (a) Photograph of the wafer, (b) Zoom on one structure. 


\section{Characterization of the device}

The quality of the micromirrors was characterized in terms of roughness and reflectivity of the $\mathrm{Cr} / \mathrm{Au}$ layers deposited on test sample. Optical profilometer using white light interferometry is used and a surface average roughness $\left(S_{a}\right)$ of $3 \mathrm{~nm}$ and a $1 \mathrm{D}$ average roughness $\left(R_{a}\right)$ of $1.7 \mathrm{~nm}$ were measured when using a cut-off wavelength of $0.25 \mathrm{~mm}$. The flatness of the micromirror is also important for the OCT application. The profile of the mirror is shown in Fig. 14 and a radius of curvature of $700 \mathrm{~mm}$ was measured by polynomial fitting of the curve. This static deformation of the reference micromirror does not affect the optical system since its maximal deformation $(52 \mathrm{~nm})$ is much smaller than the axial resolution of $6 \mu \mathrm{m}$. Finally, a high reflectivity of $97 \%$ was measured for different incidence angles at $850 \mathrm{~nm}$.

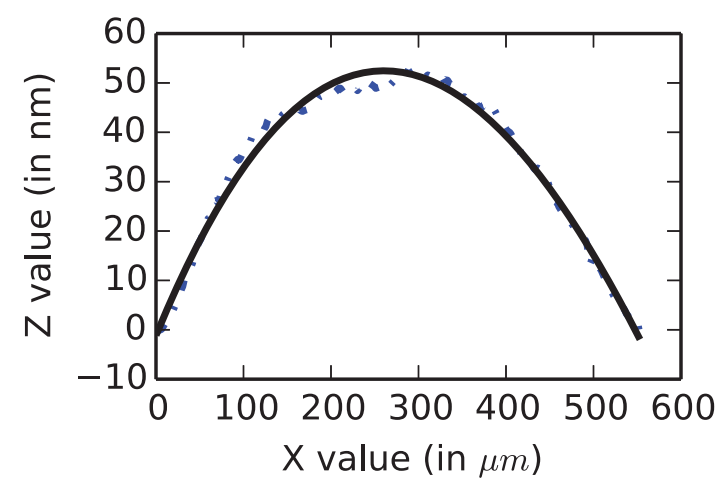

Fig. 14. Vertical deformation of the micromirror along its diagonal.

The microactuators and the microsensors were characterized at the chip level. For the characterization of individual chips, the device is glued and wire bonded to a MOEMS PCB, as in Fig. 15 (a), for further capacitance measurements and actuators driving. In order to enable optical measurements, an aluminium cap, equipped with a glass window, is crewed to the PCB (Fig. 15, (b)). A Mother PCB is also designed to facilitate the connection of different MOEMS PCBs by use of vertical pins and sockets. This solution enables the characterization of the microscanners out of the clean-room environment without the risk of contamination. (Fig. 15, (b)). This system avoid the use of probes that does not allow a small packaging system and which results in possible contamination of the chip under test.

(a)

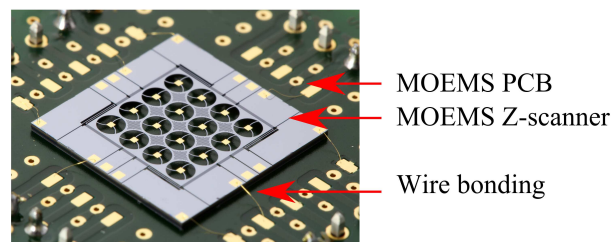

(b)

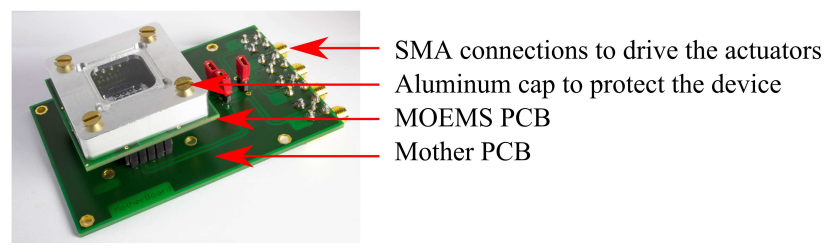

Fig. 15. Packaging of individual chips of microscanner: (a) MOEMS PCB with wire-bonded chip, (b) Complete system with MOEMS PCB connected through vertical pins into Mother PCB.

Individual chips were characterized with the MEMS Analyzer (MSA-500) from Polytec (Fig. 16). Doppler vibrometry is used for out-of plane displacement measurements. Static and dynamic measurements are realized. Note that filters are used to stabilize the signal during the measurements. Dynamic measurements consist in analysing the spectral response of the system. The excitation signal used is a sinusoidal chirp with equivalent energy for each swept frequency applied to all actuators. The first resonant frequency, 
(a)

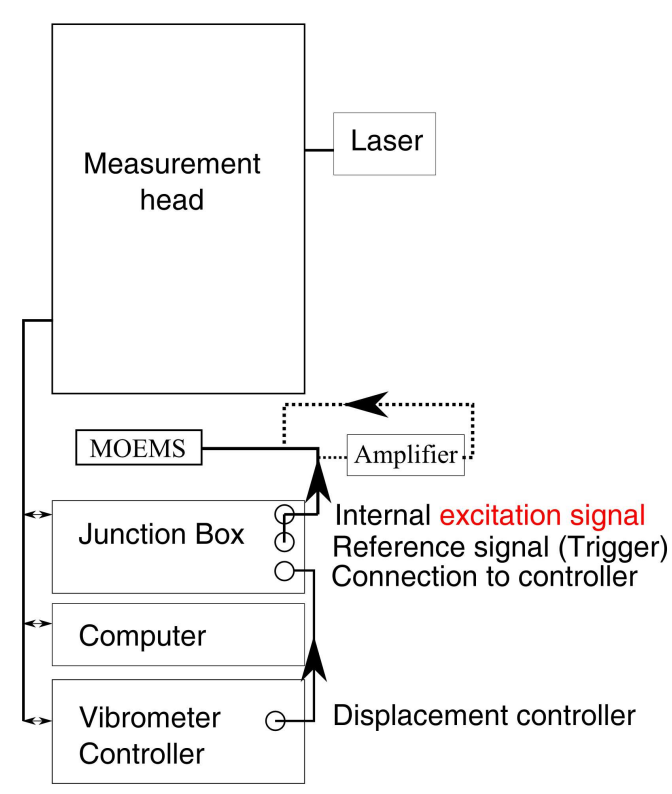

(b)

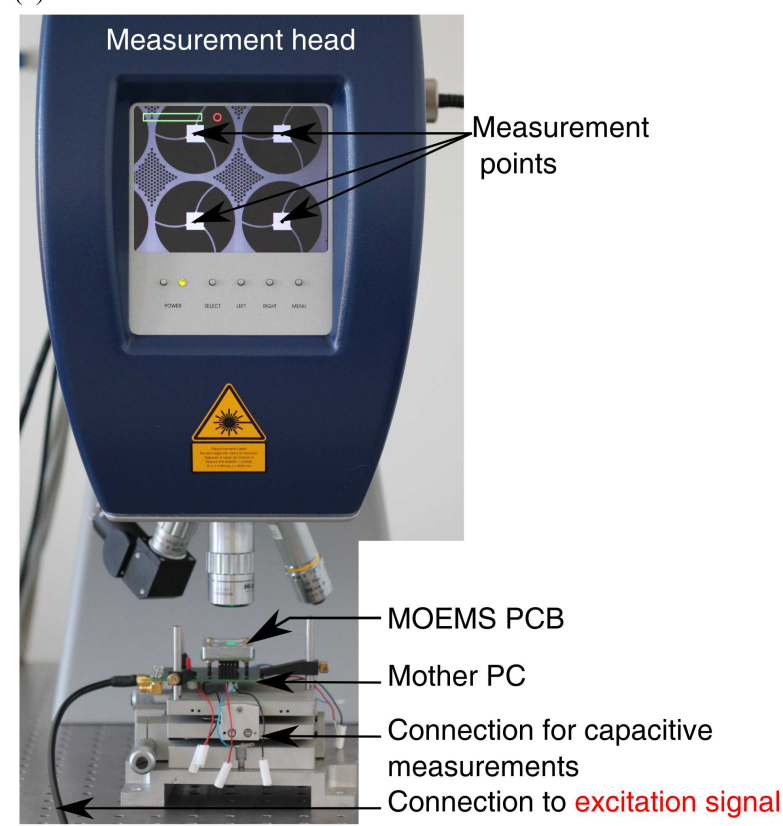

Fig. 16. MEMS analyzer setup for the displacement characterization: (a) Schematic of the connections, (b) Photograph of the setup.

which is piston mode, was measured to be $543 \mathrm{~Hz}$. The quality factor of the system was 50 based on calculations with the spectral response $(-3 \mathrm{~dB})$ and the free-oscillations characterization (exponential decrease) (respectively, Fig. 17 and 18). For such a large platform, this quality factor is relatively high compared with the $3 \times 3$ array of individually driven micromirrors presented in [6], for which the quality factor of each micromirror is 41 . The measured resonant frequency corresponds to a vertical spring stiffness of $7.2 \mathrm{~N} / \mathrm{m}$, which is coherent with the simulations, if we take into account mass $=2 \cdot 46 \cdot 10^{-6} \mathrm{~kg}$. Finally in Fig. 17, the peak corresponding to the second and third mode cannot be seen because it is too small when symmetric actuation is provided by driving of the four actuators. Then the torsional modes will not interfere when piston mode vertical actuation at the resonant frequency is performed. Static measurements were done by applying simultaneously a square signal to the 4 actuators at very low frequency $(10 \mathrm{~Hz})$ so that doppler vibrometry could be used. The static displacement was retrieved as a function of the peak-to-peak amplitude of the excitation square signal, between $0 \mathrm{~V}$ and the actuation voltage $(0-40 \mathrm{~V})$. Due to a limitation of the internal excitation signal of the MSA-500, an external amplifier was connected between the signal excitation output and the MOEMS (Fig. 16 (a)). The static displacement is plotted as a function of the applied voltage for voltages between 0 and $40 \mathrm{~V}$ in Fig. 19. The amplitude of vertical displacement was measured between 0 and approximately $2.8 \mu \mathrm{m}$, slightly depending on the gap fingers. Though, the measured static displacement curve is closer to the simulated curve obtained for $4 \mu \mathrm{m}$ gap between moving fingers. This result is consistent with the SEM measurements of the fabricated structures that show a finger gap close to $4 \mu m$.

For the phase shifting, it is important to know the displacement amplitude of all the mirrors. The dynamic displacement of the platform at resonant frequency was investigated at different points of the platform, i.e. at the position of the four micromirrors (see Fig. 2,(b)) forming a quarter of the platform (Fig. 20). The actuation voltage is set to $V_{d}=1+1 \times \sin \left(w_{0} t\right) V$. Note that the electrostatic force is proportional to the square of the voltage. Then, with the applied voltage $V_{d}$, the obtained force is a sum of a DC force, a force 

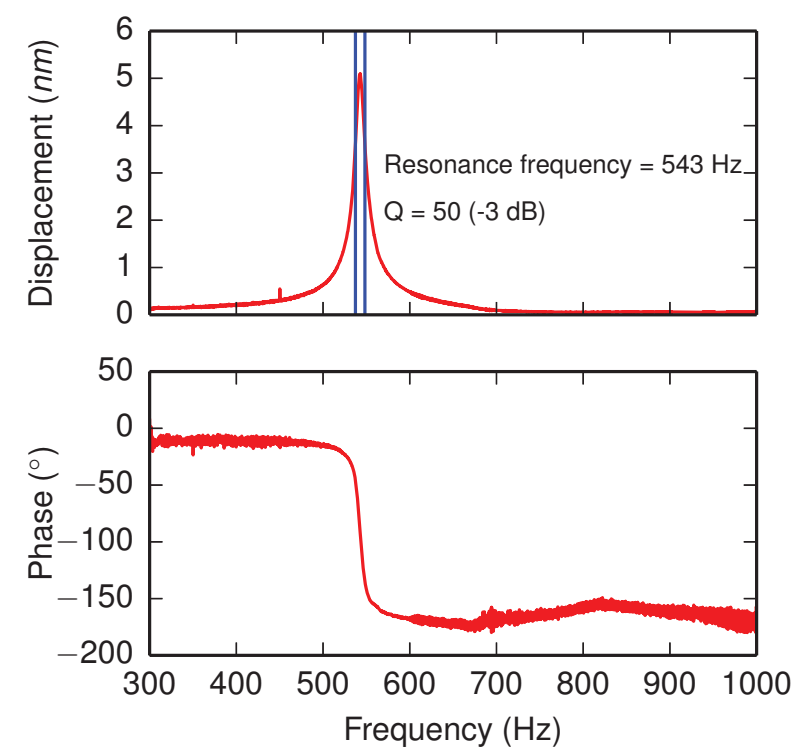

Fig. 17. Spectral response analysis of the device: (a) displacement, (b) phase.

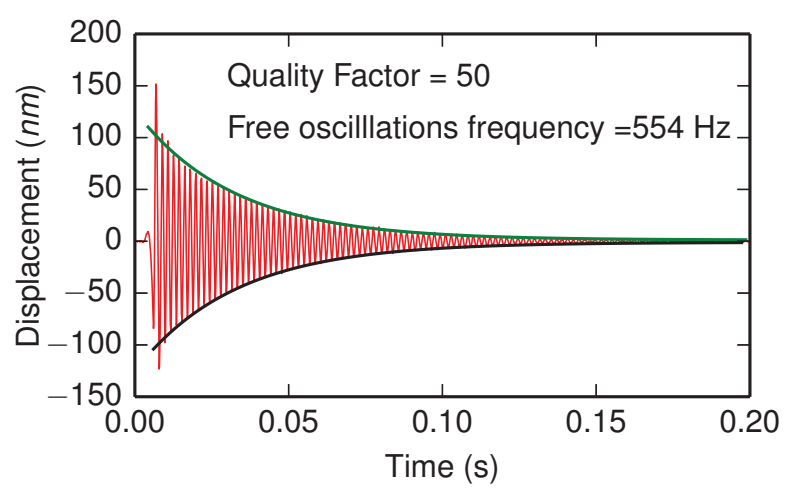

Fig. 18. Free oscillation in response to a square signal (0-5V).

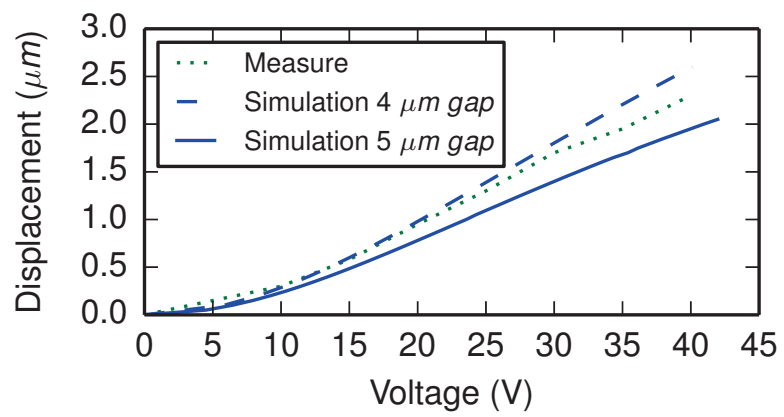

Fig. 19. Static displacement as a function of applied voltage, measured by Laser Doppler vibrometry.

proportional to $\sin \left(w_{0} t\right)$ and a force proportional to $\sin \left(2 w_{0} t+\frac{\pi}{2}\right)$. Here, the quality factor $\mathrm{Q}$ of the first mode $\left(w_{0}\right)$ is much higher so that the signal in $\sin \left(2 w_{0} t+\frac{\pi}{2}\right)$ is negligible and the dynamic displacement is synchronous with the driving voltage. Moreover, here, only dynamic displacements were measured and not 
the DC displacement. The measured displacement, much larger than the targeted one, shows the potential of this microscanner for medical imaging applications, for which small voltages are particularly important since the device is in contact with the patient. The displacement of the micromirror in the middle of the platform is $70 \mathrm{~nm}$ higher than the one next to the spring because of the vertical mechanical deformation of the suspended platform. Sasaki et al. studied the impact of the error of the displacement amplitude on the sinusoidal modulation in interferometric applications [15]. This displacement of $70 \mathrm{~nm}$ is within the tolerances presented in the article.

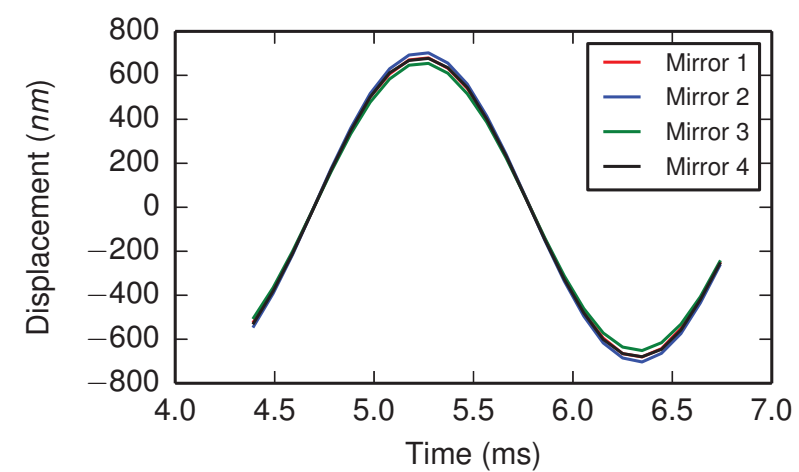

Fig. 20. Vertical displacement of the micromirror under dynamic driving voltage: (a) Mirror 1, (b) Mirror 2, (c) Mirror 3, (d) Mirror 4.

Finally, characterization of the sensing function has been performed. An impedance analyser (Agilent HP 4194A) is used to measure successively the capacitance of all the sensors as a function of applied voltage. The continuous polarization of the microactuators is obtained by an association of batteries in series $(5 \times 9 \mathrm{~V})$ to limit noise effects during the measurements. A high nominal capacitance was measured around $50 \mathrm{pF}$ with a precision of $\pm 5 f F$. This can be explained by parasitic capacitances. However, here, the variations of the capacitances are interesting to retrieve the vertical displacement. Therefore this nominal capacitance, i.e. the capacitance measured at $V_{\text {actuation }}=0 \mathrm{~V}$, which is stable during the measurement time, has been subtracted from the measured values. The measures were taken at $f=1.1 \mathrm{MHz}$, and eight measures were used to obtain each average value. The oscillation level of the impedance analyser was set to $0.5 \mathrm{~V}$. The capacitances were measured as a function of the applied voltage and then retrieved as a function of the vertical displacement using the results presented in Fig. 19. The differential capacitance of each sensor, i.e. the capacitance of Configuration 1 minus the capacitance of Configuration 2, is plotted as a function of the vertical displacement in Fig. 21. The sensitivities for each sensors correspond to the simulated sensitivity for a gap of $4 \mu \mathrm{m}$, i.e. $27 \mathrm{fF} / \mu \mathrm{m}$. One of the four sensors has a higher sensitivity of $36 \mathrm{fF} / \mu \mathrm{m}$. One explanation is that the platform is slightly tilted toward the side corresponding to this sensor creating an offset. Also a local structural defect can explain such a small increase of the sensitivity. 
(a)

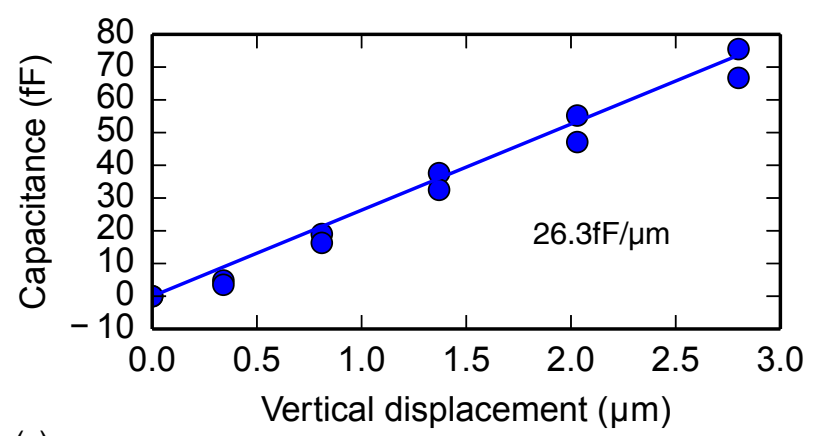

(c)

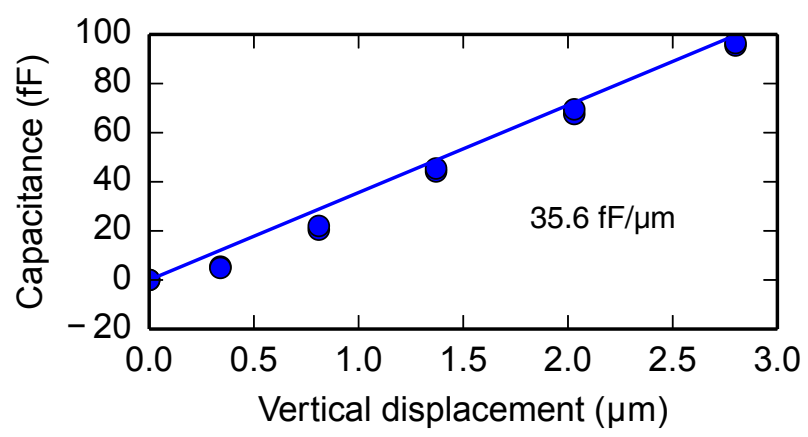

(b)

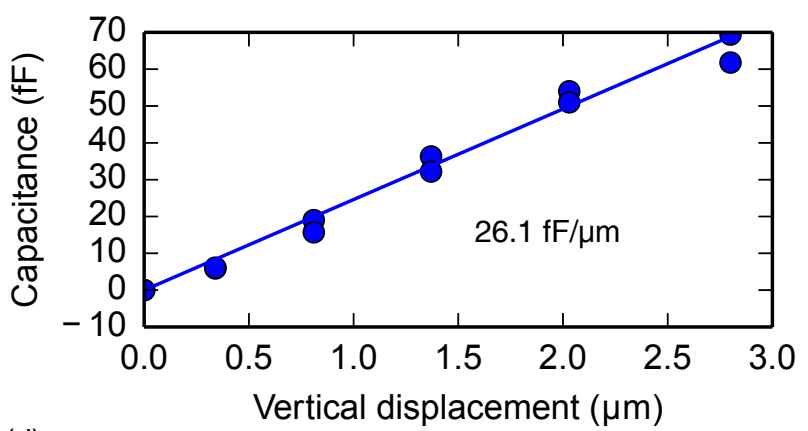

(d)

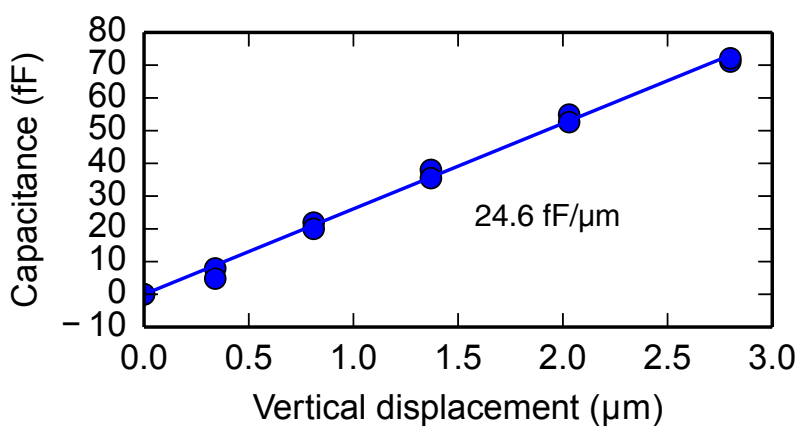

Fig. 21. Measured differential capacitance for each sensor of a chip and their corresponding sensitivity: (a) Sensor 1, (b) Sensor 2, (c) Sensor 3, (d) Sensor 4.

\section{Conclusion}

We report the design, the simulation and the fabrication of a micromirror electrostatic vertical microscanner which is a key component of a multi-channel "active" Mirau microinterferometer. Each functional part of the microscanner is developed according to the optical design of the microinterferometer in which the microscanner is to be integrated. In particular, the fabrication process is optimized and described in details for typical critical steps in MOEMS fabrication, e.g. protection of optical coatings, asymmetric vertical comb-drive etching, release of an actuated structure and chip separation. Finally, the characterization confirms that the microscanner meets the optical and mechanical targeted specifications. For the implementation of the phase modulating algorithm, the microscanner is driven sinusoidally at its resonance frequency $(\sim 500 \mathrm{~Hz})$. The use of such technique leads to a enhancement of the dynamic signal of the optical system. This miniaturized multi-channel interferometer shows the potential of MOEMS for the development of vertically integrated microsystems for applications in biomedical imaging. Future perspectives include the vertical wafer-level assembly of the microlens wafer, the micromirror wafer and the beam splitter wafer for the realization of the "active" Mirau microinterferometer. This vertical assembly is based on a silicon-glass anodic bonding. This process is potentially dangerous for the final optical quality of the micromirrors due to the high bonding temperature and for the microactuator due to the generated electrical field. Hence these steps require further work. Finally, the whole stack must be diced into individual chips which is not trivial due to the total thickness of the stack $(5 \mathrm{~mm})$.

\section{Acknowledgement}

The authors thank Gilles Bourbon for his insights and for fruitful scientific discussions. This work is financed by the collaborative project VIAMOS of the European Commission (FP7, ICT program, grant no. 
318542) and supported by the Labex Action program (ANR-11-LABX-0001-01), by the French RENATECH network, by the Collegium SMYLE and by the FEMTO-ST institute facilities.

\section{References}

1. James G Fujimoto. Optical coherence tomography for ultrahigh resolution in vivo imaging. Nat. Biotechnol., 21(11):1361-7, November 2003.

2. Dae Yu Kim, John S Werner, and Robert J Zawadzki. Comparison of phase-shifting techniques for in vivo full-range, high-speed Fourier-domain optical coherence tomography. J. Biomed. Opt., 15(October 2010):056011, 2010.

3. Li Fan, Wibool Piyawattanametha, Ming C. Wu, Aaron D. Aguirre, Paul R. Herz, Yu Chen, and James G. Fujimoto. High-resolution 3D OCT imaging with a MEMS scanning endoscope. In MOEMSMEMS Micro 85 Nanofabrication, volume 5719, pages 140-143, January 2005.

4. Abraham P Lee, Charles F. McConaghy, Gary Sommargren, Peter Krulevitch, and Eugene W Campbell. Vertical-actuated electrostatic comb drive with in situ capacitive position correction for application in phase shifting diffraction interferometry. J. Microelectromechanical Syst., 12(6):960-971, 2003.

5. Hyuck Choo, Rishi Kant, and David Garmire. Fast, MEMS-based, phase-shifting interferometer. In Solid-State Sens. Actuator Work., pages 94-95, 2006.

6. Jin-Chern Chiou, Chen-Chun Hung, Li-Jung Shieh, and Zhao-Long Tsai. Novel electrostatic MOEMS phase shifter array using CMOS-MEMS process. J. Micro/Nanolithography, MEMS MOEMS, 9:013030, 2010

7. Kentaro Oda, Hidekuni Takao, Kyohei Terao, Takaaki Suzuki, Fusao Shimokawa, Ichirou Ishimaru, and Fumikazu Oohira. Vertical comb-drive MEMS mirror with sensing function for phase-shift device. Sensors Actuators, A Phys., 181:61-69, 2012.

8. O G Karhade, F L Degertekin, and T R Kurfess. SOI-based micro scanning grating interferometers: device characterization, control and demonstration of parallel operation. J. Micromech. Microeng., 18:045007, 2008.

9. Malgorzata Kujawinska, Kay Gastinger, Michal Jozwik, Karl Henrik Haugholt, Christoph Schaeffel, and Stephan Beer. An interferometric test station for massive parallel inspection of MEMS and MOEMS. Photonics Lett. Pol., 1(2):58-60, 2009.

10. J Albero, S Bargiel, N Passilly, P Dannberg, M Stumpf, U D Zeitner, C Rousselot, K Gastinger, and C Gorecki. Micromachined array-type Mirau interferometer for parallel inspection of MEMS. J. Micromech. Microeng., 21(6):065005, June 2011.

11. Wei Xu, Alain Bosseboeuf, Fabien Parrain, and Emile Martincic. Design of a long range bidirectional MEMS scanner for a tunable 3D integrated Mirau interferometer. In Symp. Des. Test, Integr. Packag. MEMS/MOEMS, pages 1-6. IEEE, April 2014.

12. Else Gallagher, Walied Moussa, and Mark McDermott. A review of fabrication processes for vertical comb drives. Microsyst. Technol., 18(4):381-397, January 2012.

13. J. Krauter, T. Boettcher, W. Lyda, W. Osten, N. Passilly, L. Froehly, S. Bargiel, J. Albero, S. Perrin, J. Lullin, and C. Gorecki. Optical design of a vertically integrated array-type Mirau-based OCT system. In Photonics Eur., page 91320L, 2014.

14. Peng $\mathrm{Bu}$, Xiangzhao Wang, and Osami Sasaki. Full-range parallel Fourier-domain optical coherence tomography using sinusoidal phase-modulating interferometry. J. Opt. A Pure Appl. Opt., 9:422-426, 2007. 
15. O Sasaki and H Okazaki. Analysis of measurement accuracy in sinusoidal phase modulating interferometry. Appl. Opt., 25(18):3152, 1986.

16. Wei-Shan Wang, Justine Lullin, Joerg Froemel, Maik Wiemer, Sylwester Bargiel, Nicolas Passilly, Christophe Gorecki, and Thomas Gressner. Multi-wafer bonding technology for the integration of a micromachined Mirau interferometer. In Proc. SPIE, volume 1, pages 3-9, 2015.

17. Jorge Albero, Stéphane Perrin, Sylwester Bargiel, Nicolas Passilly, Maciej Baranski, Ludovic GauthierManuel, Florent Bernard, Justine Lullin, Luc Froehly, Johann Krauter, Wolfgang Osten, and Christophe Gorecki. Dense arrays of millimeter-sized glass lenses fabricated at wafer-level. Opt. Express, 23(9):11702, 2015.

18. J E Harvey and C Ftaclas. Diffraction effects of telescope secondary mirror spiders on various imagequality criteria. Appl. Opt., 34(28):6337-6349, October 1995.

19. J L Richter. Spider diffraction: a comparison of curved and straight legs. Appl. Opt., 23(12):1907-1913, June 1984.

20. Justine Lullin, Sylwester Bargiel, Emilie Courjon, Stephane Perrin, Maciej Baranski, Nicolas Passilly, and Christophe Gorecki. Monolithic integration of a glass membrane on silicon micro-actuator for micro-interferometry. In 2014 Int. Conf. Opt. MEMS Nanophotonics, pages 87-88. IEEE, August 2014.

21. Justine Lullin, Stephane Perrin, Maciej Baranski, Sylwester Bargiel, Luc Froehly, Nicolas Passilly, Jorge Albero, and Christophe Gorecki. Impact of mirror spider legs on imaging quality in Mirau microinterferometry. Opt. Lett., 40(10):2209-2212, May 2015.

22. Kieth Fedder. Simulation of Microelectromechanical Systems. PhD thesis, 1994.

23. Toshiyuki Tsuchiya and Hirofumi Funabashi. A z-axis differential capacitive SOI accelerometer with vertical comb electrodes. Sensors Actuators A Phys., 116(3):378-383, October 2004.

24. Jiankun Wang, Zhenchuan Yang, and Guizhen Yan. Silicon-on-insulator out-of-plane electrostatic actuator with in situ capacitive position sensing. J. Micro/Nanolithography, MEMS, MOEMS, 11(3):033006, July 2012. 


\title{
Electrostatic vertical microscanner for phase modulating array-type Mirau microinterferometry
}

\author{
Justine Lullin ${ }^{1, *}$, Sylwester Bargiel ${ }^{1}$, Patrice Lemoal ${ }^{1}$, Stéphane Perrin ${ }^{1}$, Jorge Albero ${ }^{1}$, Nicolas \\ Passilly $^{1}$, Luc Froehly ${ }^{1}$, Franck Lardet-Vieudrin ${ }^{1}$ and Christophe Gorecki ${ }^{1}$ \\ ${ }^{1}$ FEMTO-ST Institute (UMR CNRS 6174, UBFC), 15B Avenue des Montboucons, 25030 Besançon, \\ France \\ *justine.lullin@femto-st.fr
}

\begin{abstract}
We present a micromachined vertical scanner with a $4 \mathrm{x} 4$ array of suspended "spider-type" micromirrors which is a key component of a multi-channel "active" Mirau microinterferometer. The microscanner provides for the vertical displacement of the reference micromirrors for the implementation of phase shifting technique. Vertical comb-drive structures are employed for actuation of micromirrors (displacement range of 1 hundreds of $\mathrm{nm}$ at $500 \mathrm{~Hz}$ ) as well as for in situ capacitive displacement sensing. Integration of micro-opto-electro-mechanical microscanners in handheld interference microscope has the advantage to significantly decrease instrument size because of the small footprint of the microscanner. Particularly interesting applications are low-cost and portable microsystems for parallel inspection of biological tissues, e.g. early diagnosis of specific diseases such as skin cancers.
\end{abstract}

\section{Introduction}

The interferometric techniques are fundamental to the optical metrology due to their unique capability of non-destructive and high-accuracy measurement of a broad range of quantities. An increasing demand for the reduction of system size (portability), lower cost as well as several new metrology challenges, such as in-vivo imaging, have made the miniaturization of interferometric systems an important issue. The combination of the array-type microinterferometers and interferometry-based imaging methods enables construction of portable systems for in-vivo medical diagnostics. The swept source optical coherence tomography (SS-OCT) is a particularly interesting candidate since it allows a fast and non-invasive 3 -D reconstruction of tissue morphology within significant penetration depth $(\sim 1 \mathrm{~mm})$ and high resolution $(5-10 \mu \mathrm{m})$ [1]. Moreover, the implementation of the phase shifting interferometry (PSI) technique [2] with "active" microinterferometer allows to improve sensitivity and to remove some drawbacks of the SS-OCT technique, such as the presence of artefacts in tomography images. PSI techniques are particularly efficient to remove disturbing interferometric terms such as the mirror term, the DC term and the auto-correlation term. Then, this technique leads to an increase of the signal dynamic.

For this purpose, the micro-opto-electro-mechanical (MOEMS) approach offers not only size and cost reduction, but also the implementation of actuated micromirrors, enabling spatial scanning of the light beam in MEMS-based endoscopic probes [3] as well as precise generation of the optical phase shift. In the literature, most phase-shifters for interferometric applications were proposed based on electrostatic comb-drive actuators [4-7]. In 2003, Lee et al. were the first to propose a phase-shifter for interferometric systems based on the levitation force produced by electrostatic comb-drive with in situ parallel plate capacitive sensing [4]. 
In 2006, Choo et al. proposed a phase-shifter based on vertical electrostatic comb-drive made out of silicon. This technology presents a great advantage of simplicity [5]. Finally, Chiou et al. presented a 3x3 array of individually actuated surface-micromachined micromirrors based on the same actuation technology than in [5] for holographic data storage. The absence of position sensing in the two latter papers is a problem for the implementation of PSI algorithm. For this purpose, Oda et al. demonstrated in 2012 a phase-shifter with in situ position sensing based on silicon vertical comb technology. However, these phase-shifters are presented as one optical component but no integration strategy in the final microinterferometer is presented. In particular, MOEMS and MEMS components can be stacked using a multi-wafer vertical integration method to build the array-type MOEMS-based microinterferometers. The potential of such integration has been demonstrated recently for an electrostatically tuned grating microinterferometer for nm-scale displacement measurements [8] as well as a miniature laser Twyman-Green interferometer [9] and 5x5 array of low-coherence Mirau microinterferometers [10]. The last two devices have been developed for a massively parallel in-line inspection of MEMS. As a global strategy of integration of a microinterferometer, the Mirau configuration is well suited due to its vertical architecture and integration capability. However, this transmission-based configuration also imposes specific constraints such as a need of clear optical area around the micromirrors. Recently, the design of an "active" single channel Mirau interferometer has been proposed by Xu et al [11]. However, the fabrication and the characterisation were not presented. Finally, the proposed design did not integrate the objective lens.

In this paper, we present a Silicon-On-Insulator (SOI)-based electrostatic vertical microscanner that generates a vertical displacement of a large platform with a $4 \mathrm{x} 4$ array of reference micromirrors for the PSI-based imaging in SS-OCT system with in situ position sensing. In the following sections, we describe in details the technology of vertical comb-drive structures [12], that are employed for both actuation and sensing purposes as well as the results of static and dynamic characterization.

\section{Design and simulation}

\section{A. Integration of the microscanner in the microinteferometer}

The vertical microscanner is a part of a global optical system and must meet its specifications. The design of the optical coherence tomography system is defined in details in [13]. Figure 1 presents schematically the construction of a multi-channel $4 \times 4$ Mirau microinterferometer, in which the microscanner is implemented. The proposed microinterferometer is based on the vertical multi-wafer integration technology and consists of a doublet of plano-convex glass lenses, a microscanner wafer with array of reference micromirrors, spacer and planar beam splitter. The light is coming from the top, focused by the lens partly in the skin and partly on the reference micromirror thanks to the beam splitter. The light going back from the skin and from the mirror recombine at the beam splitter and interfere. The sum of the two light beams is recovered by the Mirau lens. The interferometric signal, recorded by a camera, is used to reconstruct a 3D image of the sample. Then the array of 16 micromirrors gives 16 3D images of the sample. The lateral dimensions of each 3D image are defined by the micromirror size and its vertical dimension is linked to the swept-source parameters. In-plane stitching is realized in order to scan a whole 3D volume of skin. In order to use phase shifting technique, the microscanner generates the vertical movement of the whole $4 \times 4$ array of reference micromirrors at a specific frequency. The applied phase modulating algorithm was proposed by $\mathrm{P}$. Bu and al. in [14]. This method is less sensitive to phase shift errors than standard phase shifting algorithm since it is based on the amplitude and frequency modulation of the light created by the sinusoidal actuation of the reference mirror. The algorithm is based on the acquisition of four interference images. Moreover, unlike classical four steps phase shifting algorithm that requires a linear motion of the phase-shifter [5], this algorithm is adapted to sinusoidal motion. The sequence of images is taken by a camera during one half of an 
actuation period, then the actuation frequency must be at least 8 times slower than the camera. Here, the frame rate of the camera is set to $4 \mathrm{kfps}$ and the actuation frequency to $500 \mathrm{~Hz}$. In order to benefit from the high quality factor of the resonant frequency, the system is designed to work at resonance. Finally, the micromirror peak-to-peak displacement amplitude is set to $2 \times \frac{2.63 \lambda}{4 \pi}$, which is the optimum value for the implementation of this algorithm as defined in [15]. The $\lambda_{0}$ and $\delta \lambda$ of the swept light source is $840 \mathrm{~nm}$ and $50 \mathrm{~nm}$, respectively. Thus, the amplitude of the micromirror displacement is $352 \mathrm{~nm}$.

The construction of the microscanner has to fulfil several requirements related to the vertical integration within the Mirau microinterferometer. First, the microscanner wafer must be positioned up-side down in the wafer stack to ensure that the reference mirror faces the beam splitter (Fig. 1). The specificity of this microscanner design is that it works in transmission, i.e. the area around the micromirror should be transparent whereas microscanners presented in the literature usually work in reflection. Moreover, microscanner wafer must be compatible with the overall integration strategy based on anodic bonding (materials, process temperature, surface quality)(Fig. 2). Finally, electrical interconnections is provided to drive the actuators and sensors by external electronics. For this purpose, holes in the spacer are placed in such a way to enable side access to the contact pads, located on the border of each chip, during wire bonding [16].

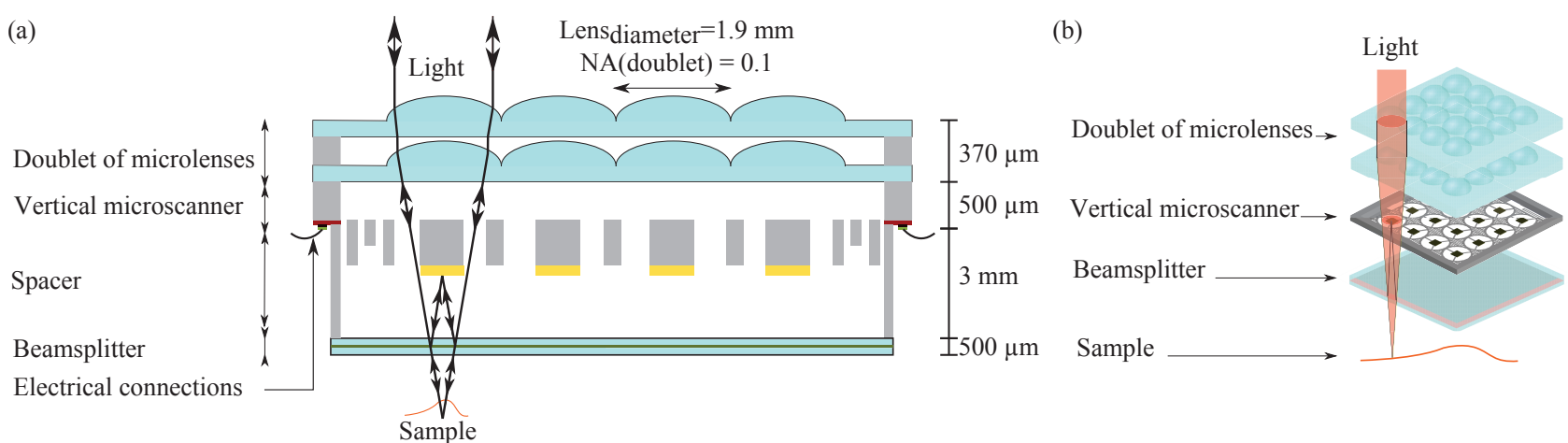

Fig. 1. Integration of the vertical microscanner within multi-channel "active" Mirau microinterferometer: (a) schematic cross-sectional view, (b) 3D expanded view.

(a)

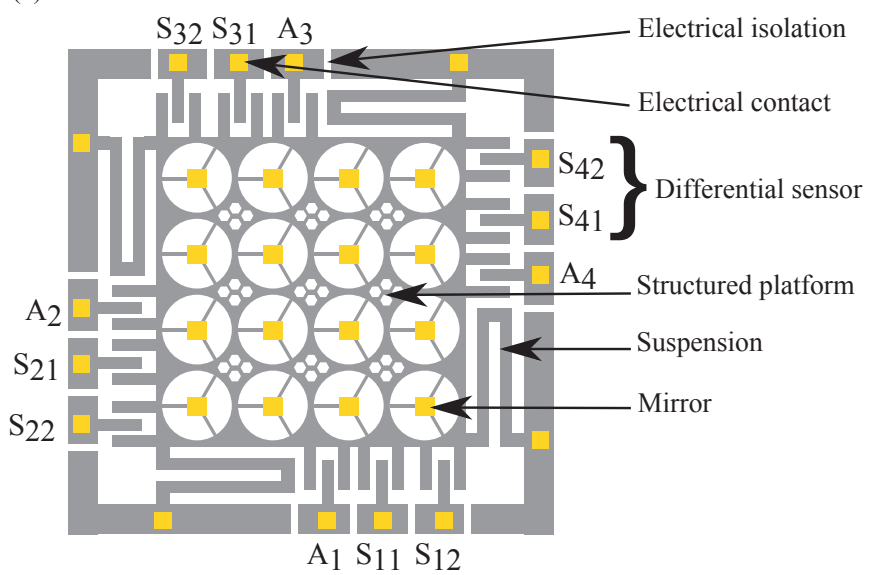

(b)

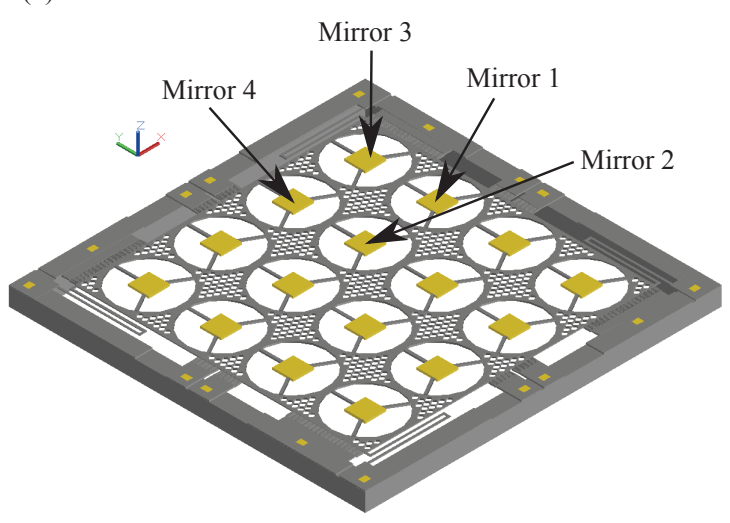

Fig. 2. Electrostatic vertical microscanner with $4 \times 4$ array of reference micromirrors: (a) Schematic top view (not in scale), (b) corresponding $3 \mathrm{D}$ view (in scale). 


\section{B. The micromirror platform}

The size and the shape of the micromirrors are defined by the optical design of the Mirau microinterferometer as a $400 \mu \mathrm{m} \times 400 \mu \mathrm{m}$ square. However, in this specific work, in-plane stitching is realized in order to reconstruct a 3D image with total lateral dimensions equal to $8 \mathrm{~mm} \times 8 \mathrm{~mm}$. Therefore, square micromirrors are preferred in order to facilitate digital processing of acquired $\mathrm{X} / \mathrm{Y}$ images. Important design issue concerns the minimization of the obscuration created by the micromirror in the center of the light beam. Moreover, the light beam is focused in the skin by the doublet of $2 \mathrm{~mm}$ diameter glass lenses, with a numerical aperture of the doublet of 0.1 [17]. The size of the square micromirror corresponds to a transmission of the mirror plane of $94 \%$ (the clear aperture around the micromirror having a diameter of $1.84 \mathrm{~mm}$ ), which is a typical value when compared to reference literatures $(70 \%-80 \%)[18,19]$. As explained before, the implementation of PSI method implies that the micromirror cannot be simply deposited on the backside of the lenses [10] but must be integrated onto an actuated platform and a clear aperture around the micromirror is needed. Two solutions are then possible, either the micromirrors are deposited on a transparent membrane [20] or they are suspended by suspensions. Integration of a glass membrane into an actuated silicon structure is a technological challenge and complicates substantially the fabrication. Therefore, the a suspended mirror is chosen. The suspensions, referred to as spider legs, obscure the light beam and create diffraction. However, it has been shown that their impact is negligible in a certain range of design parameters such as their width, number and radius of curvature [21]. According to the results of [21], the suspensions are chosen to be $50 \mu \mathrm{m}$ wide and have a radius of curvature equal to the clear aperture diameter around the micromirror. Finally, the platform is dimensioned to the array of micromirrors, vertically aligned with the lenses, forming a $8 \mathrm{~mm} \times 8 \mathrm{~mm}$ square. The platform is formed in a $40 \mu \mathrm{m}$ thick device layer of the SOI wafer and is structured with holes in order to decrease its weight. Indeed the resonant frequency of the actuated platform with micromirrors is related to the mass and the suspensions stiffness according to the following equation:

$f_{\text {resonant }}=\frac{1}{2 \pi} \times \sqrt{\frac{k}{m}}$. If $m$, the mass, is reduced, then $k$ is reduced and the vertical actuation force equal to $F_{z}=k_{z} \times z$ is minimized and so the actuation energy. A honeycomb structure is chosen for its good trade-off between mass and stiffness, preventing the possible vertical deformation of the platform. The static deformation of the mirror platform has been studied in static (Fig. 3). The maximum vertical deformation equals $140 \mathrm{~nm}$ which is much smaller than the axial resolution of the system that is $6 \mu \mathrm{m}$. Then this static deformation has no impact for the imaging system. Moreover optical simulations, made with Zemax, were done taking into account a tilt tolerance of 0.05 degree [13]. The angle, created by the vertical displacement simulated here, corresponds to a maximum micromirror angle of 0.002 degree, which is below the optical tolerance specification. 

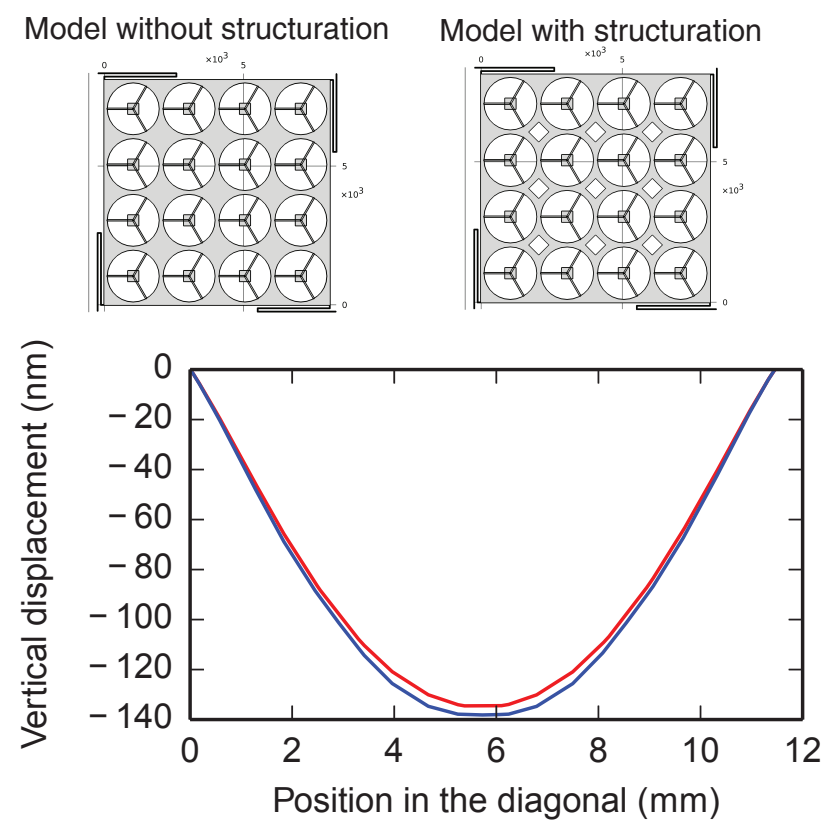

Fig. 3. Impact of structuration of the suspended platform on its vertical static deformation (diagonal direction). Two simulations are performed to analyse two limit cases in terms of structuration: one without any structuration of the platform and one with nine $700 \mu \mathrm{m}$ x $700 \mu \mathrm{m}$ square holes centered between the optical apertures. These two simulations represent two limit cases in term of structuration.

\section{The suspensions}

In order to avoid in-plane parasitic movement of actuated micromirrors, spring suspensions have been designed to be stiff in the in-plane directions. Additionally, asymmetric multi-folder springs are employed due to their small foot print (Fig. 4). The vertical stiffness is set by the targeted resonant frequency $(500 \mathrm{~Hz} \pm 50 \mathrm{~Hz})$ and the mass of the platform. The springs are placed around the platform so that the in-plane total stiffness is the same in $\mathrm{x}$ and $\mathrm{y}$ directions. In the following, silicon is modelled as an isotropic material which Young Modulus is equal to $170 \mathrm{GPa}$ and Poisson's ratio 0.26. However it has been checked that simulation results with an anisotropic and an isotropic hypothesis are very close.

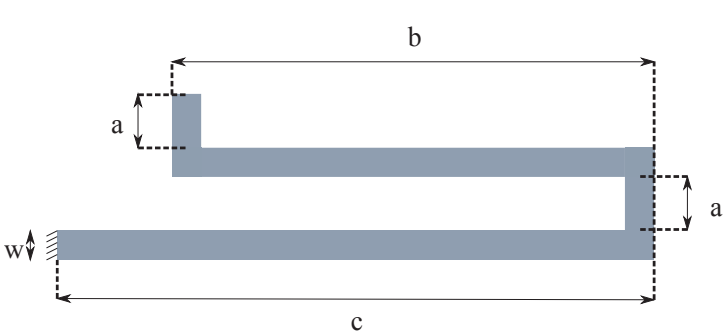

Fig. 4. Asymmetric springs dimensions.

\begin{tabular}{c|l|c} 
Symbol & Description & Value \\
\hline $\mathrm{w}$ & Beam width & $30 \mu \mathrm{m}$ \\
$\mathrm{t}$ & Beam thickness & $40 \mu \mathrm{m}$ \\
$l_{1}=a$ & Beam 1 length & $90 \mu \mathrm{m}$ \\
$l_{2}=b$ & Beam 2 length & $2610 \mu \mathrm{m}$ \\
$l_{3}=a$ & Beam 3 length & $90 \mu \mathrm{m}$ \\
$l_{4}=c$ & Beam 4 length & $2820 \mu \mathrm{m}$
\end{tabular}

Table 1. Design parameter of the asymmetric springs.

Simulations using Comsol and theoretical calculations have been performed in order to design the spring according to the specifications (Table 1). Theoretical values have been retrieved using the energy method. This method is well detailed in [22] and is based on Castigliano's second theorem. To use this method, the serpentine spring is decomposed in 4 straight beams that are analysed. The total strain energy of the linearly elastic structure is calculated as follows: 


$$
U=\frac{1}{2}\left(\sum_{i=1}^{n} \int_{0}^{l_{i}} \frac{M_{i}^{2}}{E I_{i}} d \epsilon+\sum_{i=1}^{n} \int_{0}^{l_{i}} \frac{T_{i}^{2}}{G J_{i}} d \epsilon\right)
$$

where $l_{i}$ is the length of beam,$M_{i}$ is the Moment of beam,$T_{i}$ is the Torsion of beam $I_{i}$ is the Moment of Inertia of beam,$J_{i}$ is the Torsion constant of beam ${ }_{i}$ and $\mathrm{G}$ and E are respectively the shear modulus and the Young modulus of the material.

For a rectangle cross-section, $I=\frac{w t^{3}}{12}$ and $J=\frac{1}{3} w^{3} t\left(1-\frac{192 w}{\pi^{5} t} \sum_{i=1, i \text { odd }}^{\infty} \frac{1}{i^{5}} \tanh \left(\frac{i \pi t}{2 w}\right)\right)$.

The moment and the torsion of each beam, created by $M_{0}, T_{0}$ and $F_{z}$ are defined as follows:

$$
\begin{aligned}
& M_{1}=M_{0}-F_{z} \epsilon \\
& M_{2}=T_{0}+F z \epsilon \\
& M_{3}=M_{0}-F_{z}\left(\epsilon+l_{1}\right) \\
& M_{4}=F_{z} *\left(l_{2}-\epsilon\right)+T_{0}
\end{aligned}
$$

$$
\begin{aligned}
& T_{1}=T_{0} \\
& T_{2}=M_{0}-F_{z} l_{1} \\
& T_{3}=F_{z} l_{2}+T_{0} \\
& T_{4}=M_{0}-l_{1} F_{z}-l_{3} F_{z}
\end{aligned}
$$

With the conditions:

$$
\frac{\partial U}{\partial M_{0}}=0, \frac{\partial U}{\partial T_{0}}=0 \text { and } \frac{\partial U}{\partial F_{z}}=\delta z
$$

and $l_{1}=l_{3}=a, l_{2}=b$ and $l_{4}=c$, one obtains,

$$
\begin{gathered}
k_{z}=\frac{F_{z}}{\delta z}=\frac{12 E G I J(2 E I a+G J(b+c))(E I(b+c)+2 G J a)}{S U M} \\
S U M=12 E^{3} I^{3} a^{2} b\left(2 a c+b^{2}+b c\right) \\
+4 E^{2} G I^{2} J a\left(4 a^{3} b+16 a^{3} c+6 a^{2} b^{2}+3 a b^{2} c+3 a b c^{2}+2 b^{4}+5 b^{3} c-b c^{3}+2 c^{4}\right) \\
+E G^{2} I J^{2} \quad\left(32 a^{5}+8 a^{3} b^{2}+40 a^{3} b c+32 a^{3} c^{2}+16 a^{2} b^{3}+24 a^{2} b^{2} c-24 a^{2} b c^{2}\right. \\
\left.+16 a^{2} c^{3}+b^{5}+5 b^{4} c-2 b^{3} c^{2}-2 b^{2} c^{3}+5 b c^{4}+c^{5}\right) \\
\left.+2 G^{3} J^{3} a \quad\left(8 a^{3} b+8 a^{3} c+b^{4}+4 b^{3} c-6 b^{2} c^{2}+4 b c^{3}+c^{4}\right)\right)
\end{gathered}
$$

Following the same method, we calculate $k_{x}$ and $k_{y}$. Table 2 summarizes the obtained results.

The mode shapes of the actuated platform are simulated to confirm stiffness calculations. The first mode is confirmed to be the piston mode at $527 \mathrm{~Hz}$, whereas the second mode and third mode are torsion modes at $840 \mathrm{~Hz}$ (Fig. 5). 
Table 2. Calculated and simulated stiffness of the suspension. The calculated mass $m$ of the suspended platform is equal to $2 \cdot 46 \cdot 10^{-6} \mathrm{~kg}$ which corresponds to a frequency of $530 \mathrm{~Hz}(533 \mathrm{~Hz})$.

Mode 1

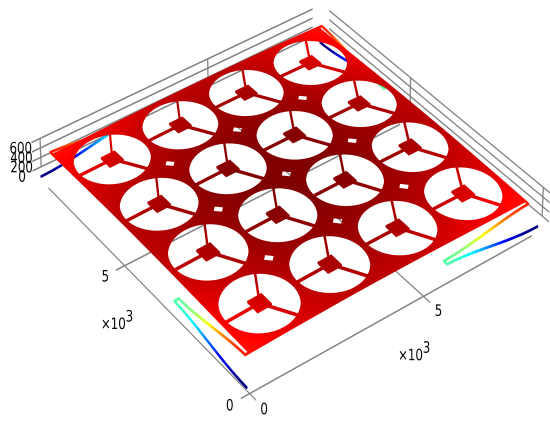

Mode 2

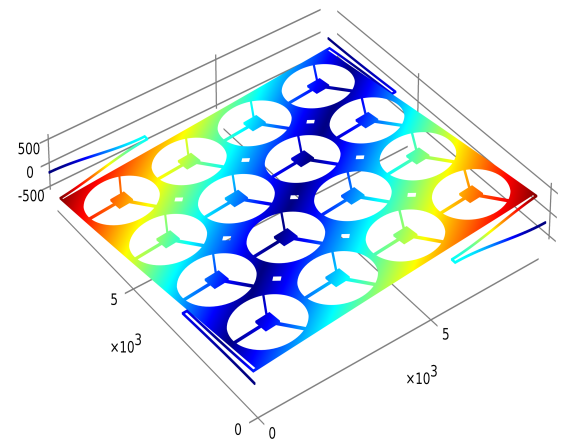

Mode 3

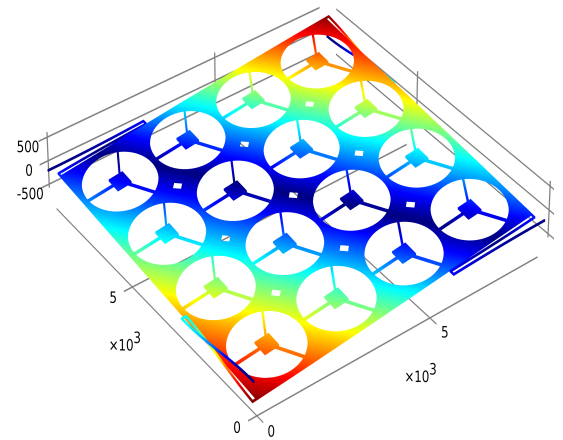

Fig. 5. Simulation of the three first natural modes of the vertical microscanner.

\section{The actuator and sensor}

Actuating and sensing are the core functions of the device. In order to use the phase shifting algorithm, it is necessary to know the displacement amplitude of the micromirrors. Therefore, a capacitive sensor is also integrated to the structure. Both the vertical actuators and sensors are based on the electrostatic field created by vertical comb fingers (Fig. 6). This technology has been intensively used due to its relative easiness of implementation in silicon-based MEMS [7,23,24]. Indeed, combination of SOI substrates and Deep Reactive Ion Etching (DRIE) allows fabrication of both the micromechanical structures as well as the electrostatic actuators and sensors.

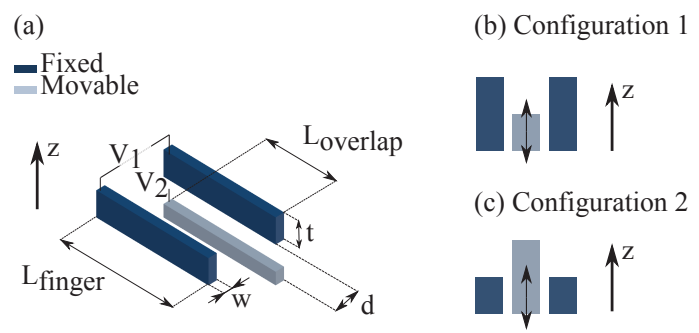

Fig. 6. Assymetrical comb finger design : (a) Dimensions design, (b) Configuration 1, c) Configuration 2. 
Actuator The vertical electrostatic force is created by a change of capacitance in the vertical direction. This is possible when a vertical asymmetry exists in the design of the comb fingers (Fig. 6, (b)). If parallel plate capacitances are used to create lateral electrostatic actuator, here the fringing capacitances are responsible for the actuation. The electrostatic force, between two opposite fingers, is proportional to the derivative of the capacitance between these fingers according to the following relationship:

$$
F_{e l}=\frac{1}{2} \frac{\partial C}{\partial z} V^{2}
$$

where $C$ is the capacitance and $\mathrm{V}$ is the potential between the two fingers.

Sensor The sensing function is created by differential capacitance. It has been shown that designing two specific types of comb fingers (Fig. 6), their differential capacitance is linear as a function of the only vertical displacement of the comb fingers [23]. When the movable finger moves up, the capacitance mainly changes for the Configuration 2 (Fig. 6, c)) whereas if the movable finger moves down then the capacitance mainly changes for the configuration 1 (Fig. 6, (b)). This method is used to design the four differential sensors, each composed of one set of fingers with the Configuration 1 and another with Configuration 2, that are placed around the movable platform as shown in Fig. 2.

Simulations Simulations of the capacitance have been performed with 3D FEM simulation using Intellisuite to study both the actuation and the sensing functions since they are based on the same physical phenomena. A model composed of one movable finger facing two fixed fingers were design. The ThermoElectro-Mechanical module was used. A MacroModelExtraction/Capacitance vs. displacement analysis and a Static/Electro Thermo Relaxation analysis were chosen to process the capacitance change and the static displacement of the platform as a function of an applied voltage between fixed and moving parts. Then Figure 7 shows the capacitance change for each of comb finger configurations with single movable finger versus the platform displacement as well as their differential capacitance.

On the one hand, linear fitting of the differential curve allows to determine the sensitivity of one couple of fingers $0.64 \mathrm{fF} / \mu \mathrm{m}$ and then calculate the sensitivity of a differential sensor, made of 34 couples of fingers, to be $22 \mathrm{fF} / \mu \mathrm{m}$. The same simulation has been performed for a gap equal to $4 \mu \mathrm{m}$ and a sensitivity of 0.81 $\mathrm{fF} / \mu \mathrm{m}$, corresponding to a total sensitivity of $27 \mathrm{fF} / \mu \mathrm{m}$ for one differential sensor, has been obtained. It is worth to note that the differential capacitance in the range of some $\mathrm{fF}$ has to be measured for required by PSI displacement amplitude.

On the other hand, the static displacement of the platform as a function of the applied voltage was simulated, based on a capacitance change simulation, for two finger gaps (4 and $5 \mu \mathrm{m})$, and compared to the experimentally obtained values (Fig.19).

The results of the capacitive simulation are coherent with the literature results (Sensitivity=0.66 $\mathrm{fF} / \mu \mathrm{m}$ and $0.68 \mathrm{fF} / \mu \mathrm{m}$ respectively in [24] and in [7] for similar parameters and technology) and are used to chose the design parameters of the comb fingers (Table 3 ). 


\begin{tabular}{c|l|c} 
Symbol & Description & Value \\
\hline$L_{\text {finger }}$ & Finger length & $200 \mu \mathrm{m}$ \\
$L_{\text {overlap }}$ & Finger overlap & $190 \mu \mathrm{m}$ \\
$\mathrm{d}$ & Gap & $5 \mu \mathrm{m}$ \\
$t$ & Finger thickness & $40 \mu \mathrm{m}$ \\
$w$ & Finger width & $10 \mu \mathrm{m}$ \\
$N_{\text {actuator }}$ & Total movable finger number & 400 \\
$N_{\text {sensor }}$ & Total movable finger number per sensor type & 136
\end{tabular}

Table 3. Design parameter of the asymmetric comb finger

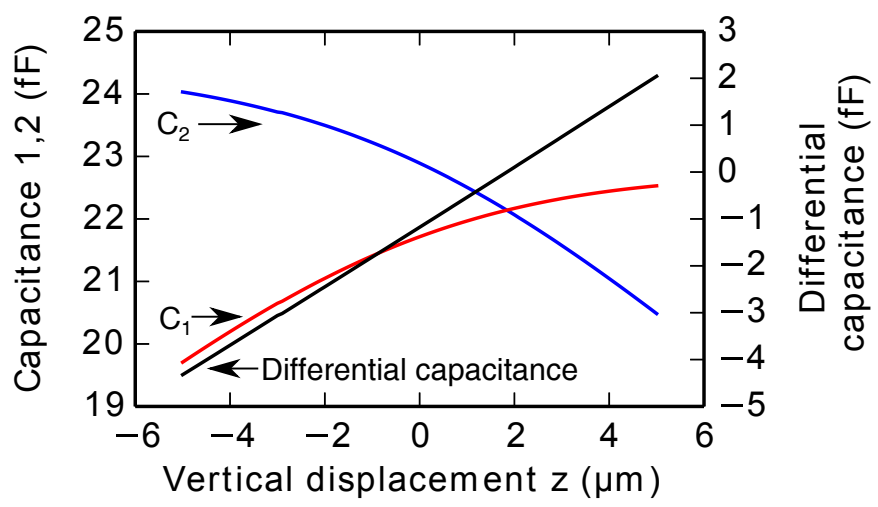

Fig. 7. Capacitance versus displacement of Configuration 1 and Configuration 2 and differential capacitance for one movable finger facing two fixed fingers. Linear fitting of the differential capacitance gives a sensitivity of $0.64 \mathrm{fF} / \mu \mathrm{m}$.

\section{Fabrication of the device}

The technology of vertical scanner is based on double-side processing of SOI wafer using DRIE method. The SOI substrate has been chosen since it ensures well-controlled thickness of device layer $(40 \pm 0.5 \mu \mathrm{m})$, in which the designed micromechanical structures will be formed. Indeed, the thickness parameter is of great importance for vertically actuated structures because of the spring stiffness sensitivity and the necessity to control a vertical offset between the comb fingers.

The first stage of the scanner fabrication, i.e. structuration of device layer, depends directly on the fabrication method of asymmetric comb fingers. In this work, it is realized by a two-steps DRIE process (Fig. 8). First, two mask layers, one in a silicon dioxide $(\mathrm{t}=0.8 \mu \mathrm{m})$ and the other in a photoresist $(\mathrm{t}$ $=2.5 \mu \mathrm{m}$ ), are formed on the device layer in two subsequent photolithography procedures (Fig. 8, steps (a)). The alignment precision achieved was $\pm 0.75 \mu \mathrm{m}$. These mask patterns, corresponding to the two different sets of the facing fingers, are used in the first DRIE process to etch all comb fingers down to the Buried Oxide $(\mathrm{BOX})$ layer $\left(t_{d l}=40 \mu \mathrm{m}\right)$. Simultaneously, other micromechanical structures, i.e. the springs, the platform and the mirrors, are also realized. After stripping the resist mask, one set of comb fingers is still protected by $\mathrm{SiO}_{2}$ mask, whereas the other is exposed to the etching plasma during the second DRIE process. In consequence, the thickness of unprotected fingers is reduced by half $\left(\frac{1}{2} t_{d l}=20\right.$ $\mu m$ ), which creates a vertical asymmetry between the facing comb fingers. This second DRIE process, realized with an ICP DRIE machine (Pegasus Rapier SPTS), has been optimized to prevent the notching effect due to the BOX. The actual gap between finger was measured to be $4 \mu \mathrm{m}$, instead of the targeted $5 \mu \mathrm{m}$, because of an overestimated under-etching during masks creation and silicon etching. Finally, an 
(a)

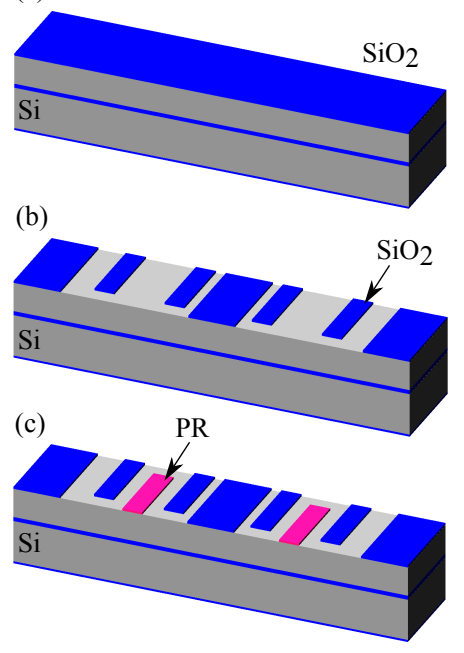

(d)

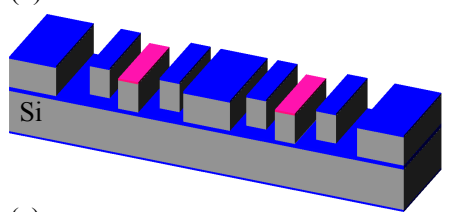

(e)

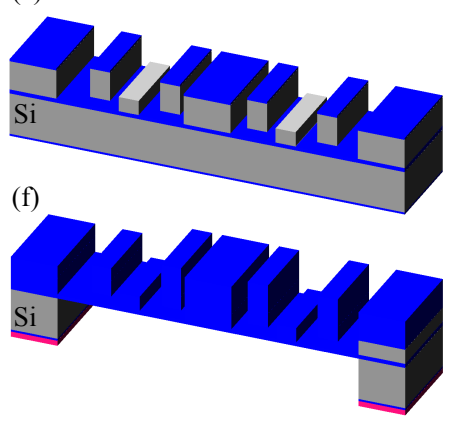

(g)

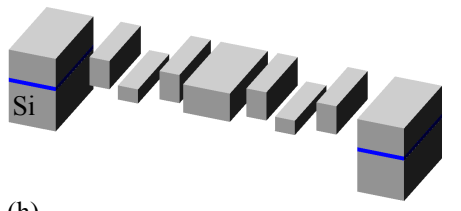

(h)

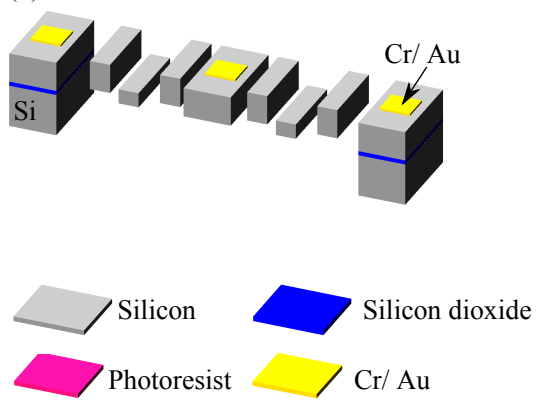

Fig. 8. Flow chart of the fabrication process: (a) oxydation of SOI wafer $(800 \mathrm{~nm})$, (b) Photolithography and ICP DRIE for structuration of $\mathrm{SiO}_{2}$ layer (Mask 1), c) Photolithography of S1828 photoresist layer (Mask 2), d) First DRIE, e) Removal of Mask 2 and Second DRIE, f) Oxidation of the wafer (200 nm) to protect front side during back side release followed by back side DRIE with thick photoresist mask, g) Vapor HF etching of $\mathrm{SiO}_{2}$ layers and BOX, h) Deposition of $\mathrm{Cr} / \mathrm{Au}(10 \mathrm{~nm} / 150 \mathrm{~nm})$ by sputtering though physical mask.

important drawback of this step is linked to its "maskless" character. Realization of classic Bosch process leads in this case to the creation of thin silicon "walls" at the edges of the processed fingers (Fig. 9 B.). This phenomena has already been observed in [23] where a passivation layer was deposited before RIE etching step. One of the possible explanations is that during this second DRIE step, the teflon-like polymer that is deposited on the finger side-walls, has finally no more silicon support and become a mask during the following etching cycle by falling down on the structure. Another explanation would be a micromasking effect, related to high selectivity of silicon etching in SPTS system towards polymer $(S=150)$ and oxide $(S=250)$ masks as well as the deformation of local electric field due to sharp edges of fingers. In [23], solutions were suggested but not tested. Here, the proposed solution relies on increased time of $\mathrm{SF}_{6}$ etching cycle of Bosch procedure in order to systematically under-etch the remaining thin silicon wall (Fig. 9).

The second stage of the scanner fabrication deals with the release of the structure. This part is always very sensitive when it comes to active structure. Here, the back side of the device, i.e. the handle layer $\left(t_{h l}=500\right.$ $\mu m$ ), must be completely etched to allow light transmission through the platform, around the micromirrors. This etching step is realized through a $15 \mu \mathrm{m}$ thick AZ9260 resist mask deposited on the $\mathrm{SiO}_{2}$ layer. Note that $\mathrm{SiO}_{2}$ etching step should be preferably done with successive short etching times to limit an elevation of the temperature that leads to difficulties in removing the resist. Moreover, it has been found that some cracks appear in the BOX when it is reached by the etching plasma. The consequence is the penetration of the etching plasma through the BOX layer and then the partial etching of the device layer, destroying the structure (Figure 10). Therefore, a thin thermal silicon dioxide layer $(t=150 \mathrm{~nm})$ is thermally grown to protect the front side during the back-side etching. Finally, the release of the platform is done by etching of the silicon dioxide layers in vapour HF (Fig. 8, step (f)). In order to ensure the best possible quality of micromirror surface as well as contact pads, the deposition of the metal layer is performed as a last step. For the fabrication of micromirrors, the sputtering of $\mathrm{Cr} / \mathrm{Au}$ layer $(10 \mathrm{~nm} / 150 \mathrm{~nm})$ is realized through a physical 
A.

(a)

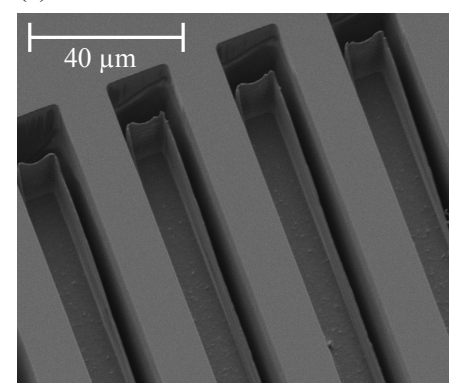

(b)

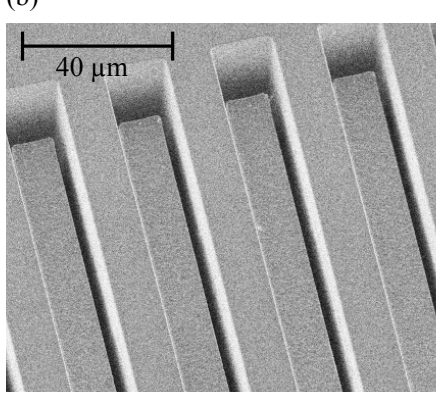

B.

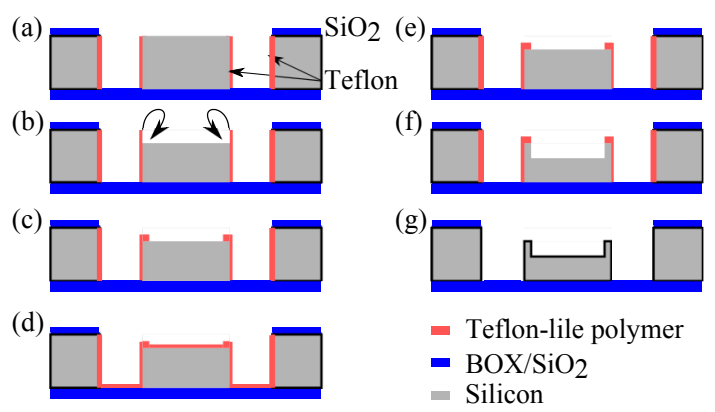

Fig. 9. SEM image of fabricated comb fingers. A) SEM images : (a) Creation of silicon "wall" during second DRIE where there is no masking layer (Fig. 8 step e)) (b) Improved DRIE process to limit silicon "wall" creation. B) Schematic of the formation of silicon walls during DRIE process : (a) Deposition of teflon everywhere and isotropic etching of the teflon on horizontal surfaces, (b) Silicon etching, with formation of free teflon walls, (c) Falling of teflon walls on the silicon, d) Deposition of teflon, (e) Isotropic etching of teflon on horizontal surfaces, some teflon is left, (f) Silicon etching with teflon residue on horizontal surfaces creating silicon walls, (g) Cleaning of the wafer.

mask, aligned thanks to bonding tools (Fig. 11). For this purpose, both the microscanner and physical wafers are aligned directly in a EVG bonding tool using EVG620 mask alignment system, and then brought into contact in EVG501 wafer bonder. The alignment is maintained in the sputtering machine thanks to an adhesive teflon tape. The physical mask has been designed in order to minimize the tolerance sensitivity.

A.

(a)

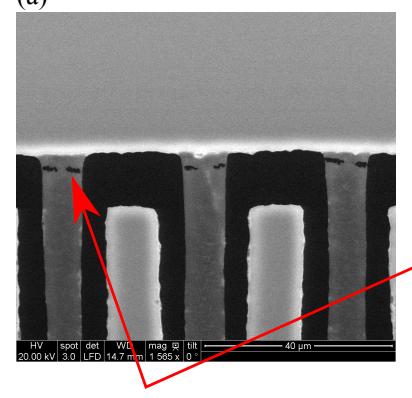

Fingers etched by the back side (b)

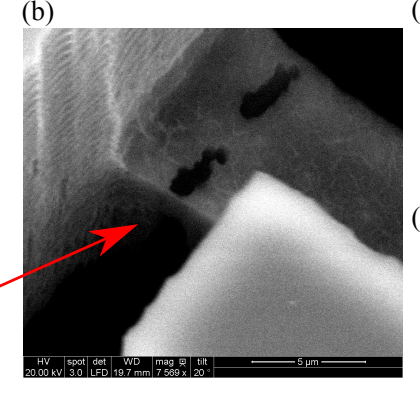

B.

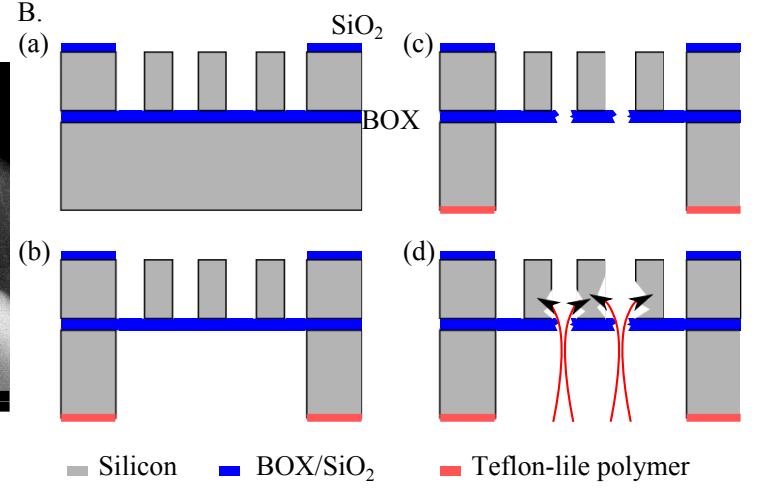

Fig. 10. SEM image after back side DRIE. A : SEM images (a) Comb fingers that are partially etched from the back side, (b) Zoom on one finger. B : Schematic of the etching of comb fingers by the back side through BOX cracks : (a) Comb fingers SOI wafer, (b) Back side DRIE etching down to the BOX, (c) Creation of cracks in the BOX, (d) Penetration of etching plasma through the BOX from the back side to the front side of the wafer resulting in comb fingers destruction.

Two strategies of fabrication were implemented: the first to enable the separation of individual chips from the wafer and thus facilitate the characterization (Fig. 12) and the second for the future wafer-level integration within the Mirau microinterferometer (Fig. 13). With the first design, individual chips were separated using UV tape to maintain them while manually cutting small silicon bridges and sucking up the dust using vacuum system (Fig. 12). Finally, short exposure of tape to UV light allows gentle releasing the chips. One wafer with this "chip" design was fabricated without optimization and a yield of $71 \%$ was obtained. For 
(a)

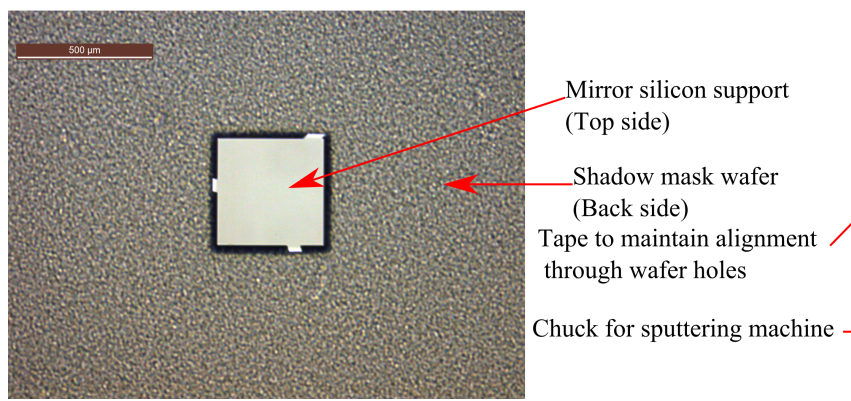

(b)

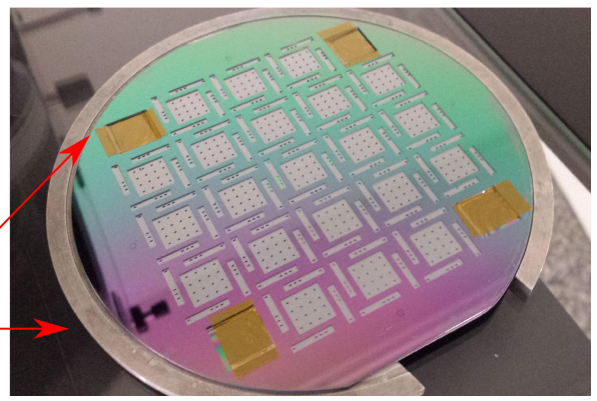

Fig. 11. Deposition of $\mathrm{Cr} / \mathrm{Au}$ layer on micromirrors and pads using shadow mask wafer: (a) image of the shadow wafer aligned with the SOI wafer showing the mirror silicon support, (b) a global view of the aligned wafers (top : shadow mask, bottom : SOI wafer) ready for the mirror sputtering.

the second design, three wafers were fabricated to test the vertical integration of the microscanner with the glass microlenses wafer and the beam splitter. The yield of fabrication of these wafers was $100 \%$ (Fig. 13 (a)).

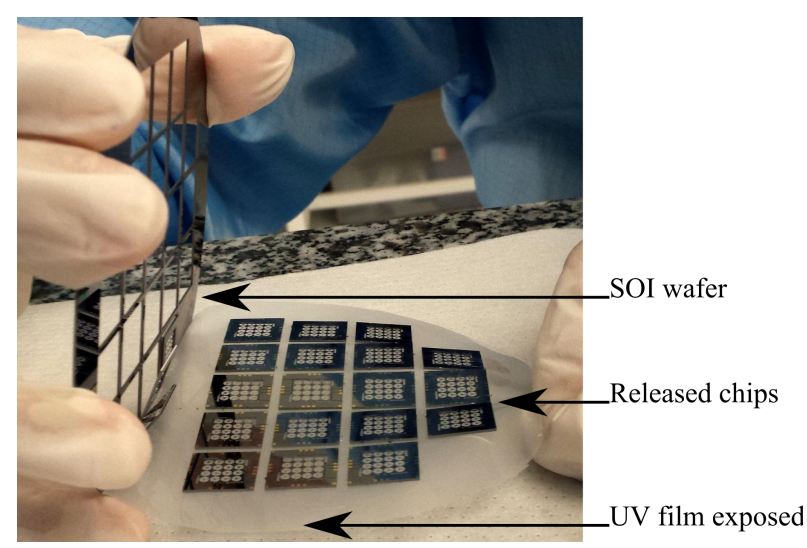

Fig. 12. Design 1: Individual chips separated from the wafer for characterization.

(a)

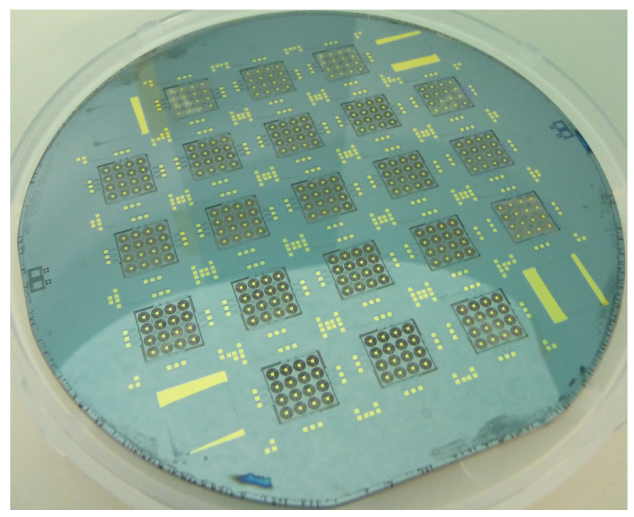

(b)

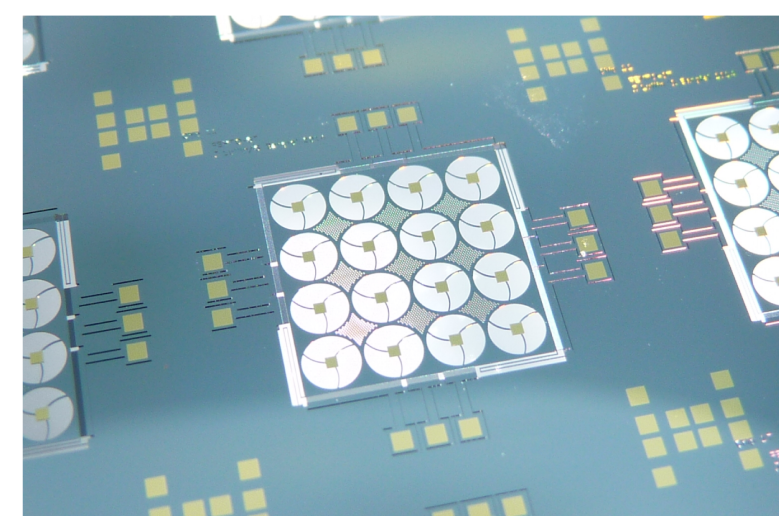

Fig. 13. Design 2: Released microscanner that are kept at wafer level for future vertical wafer-level integration: (a) Photograph of the wafer, (b) Zoom on one structure. 


\section{Characterization of the device}

The quality of the micromirrors was characterized in terms of roughness and reflectivity of the $\mathrm{Cr} / \mathrm{Au}$ layers deposited on test sample. Optical profilometer using white light interferometry is used and a surface average roughness $\left(S_{a}\right)$ of $3 \mathrm{~nm}$ and a $1 \mathrm{D}$ average roughness $\left(R_{a}\right)$ of $1.7 \mathrm{~nm}$ were measured when using a cut-off wavelength of $0.25 \mathrm{~mm}$. The flatness of the micromirror is also important for the OCT application. The profile of the mirror is shown in Fig. 14 and a radius of curvature of $700 \mathrm{~mm}$ was measured by polynomial fitting of the curve. This static deformation of the reference micromirror does not affect the optical system since its maximal deformation $(52 \mathrm{~nm})$ is much smaller than the axial resolution of $6 \mu \mathrm{m}$. Finally, a high reflectivity of $97 \%$ was measured for different incidence angles at $850 \mathrm{~nm}$.

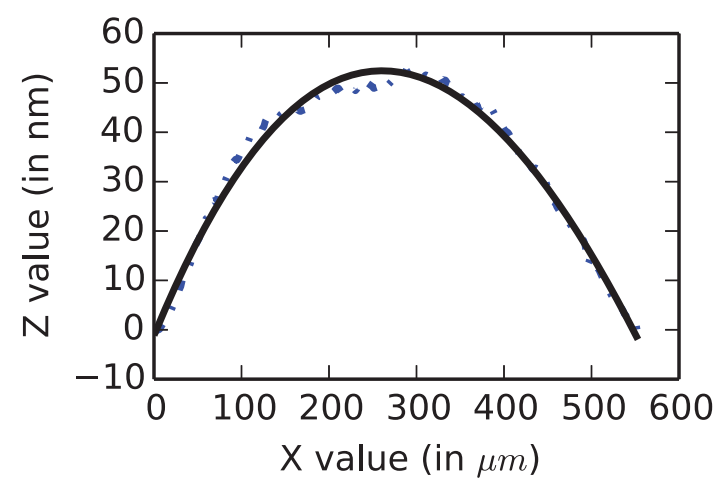

Fig. 14. Vertical deformation of the micromirror along its diagonal.

The microactuators and the microsensors were characterized at the chip level. For the characterization of individual chips, the device is glued and wire bonded to a MOEMS PCB, as in Fig. 15 (a), for further capacitance measurements and actuators driving. In order to enable optical measurements, an aluminium cap, equipped with a glass window, is crewed to the PCB (Fig. 15, (b)). A Mother PCB is also designed to facilitate the connection of different MOEMS PCBs by use of vertical pins and sockets. This solution enables the characterization of the microscanners out of the clean-room environment without the risk of contamination. (Fig. 15, (b)). This system avoid the use of probes that does not allow a small packaging system and which results in possible contamination of the chip under test.

(a)

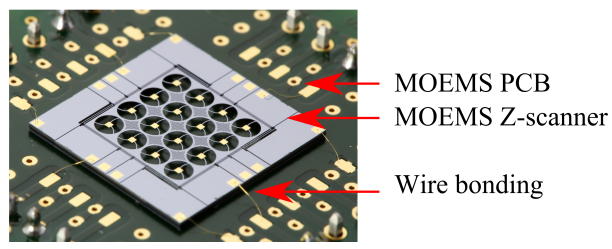

(b)

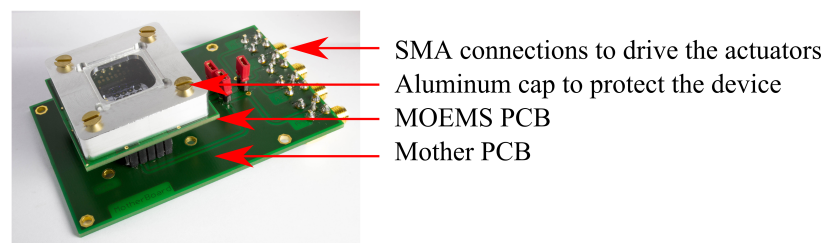

Fig. 15. Packaging of individual chips of microscanner: (a) MOEMS PCB with wire-bonded chip, (b) Complete system with MOEMS PCB connected through vertical pins into Mother PCB.

Individual chips were characterized with the MEMS Analyzer (MSA-500) from Polytec (Fig. 16). Doppler vibrometry is used for out-of plane displacement measurements. Static and dynamic measurements are realized. Note that filters are used to stabilize the signal during the measurements. Dynamic measurements consist in analysing the spectral response of the system. The excitation signal used is a sinusoidal chirp with equivalent energy for each swept frequency applied to all actuators. The first resonant frequency, 
(a)

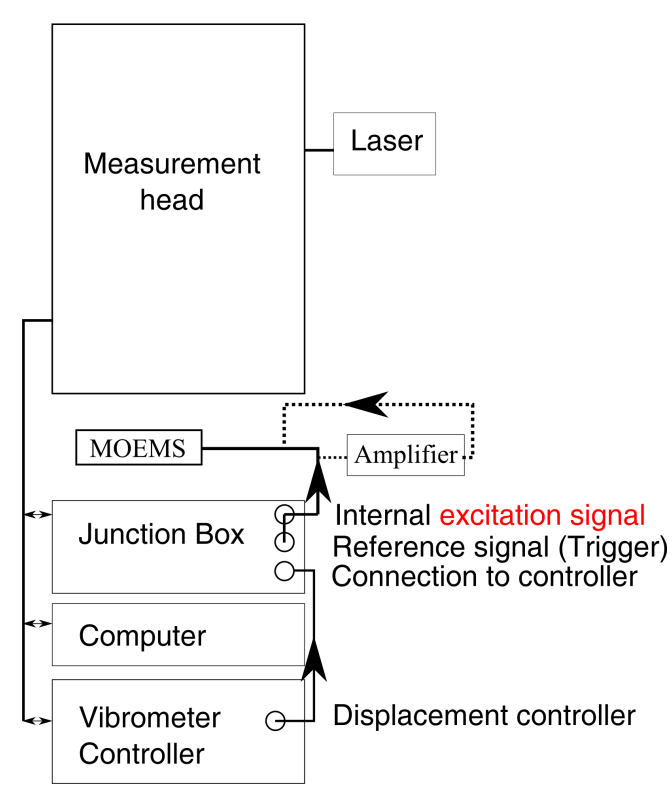

(b)

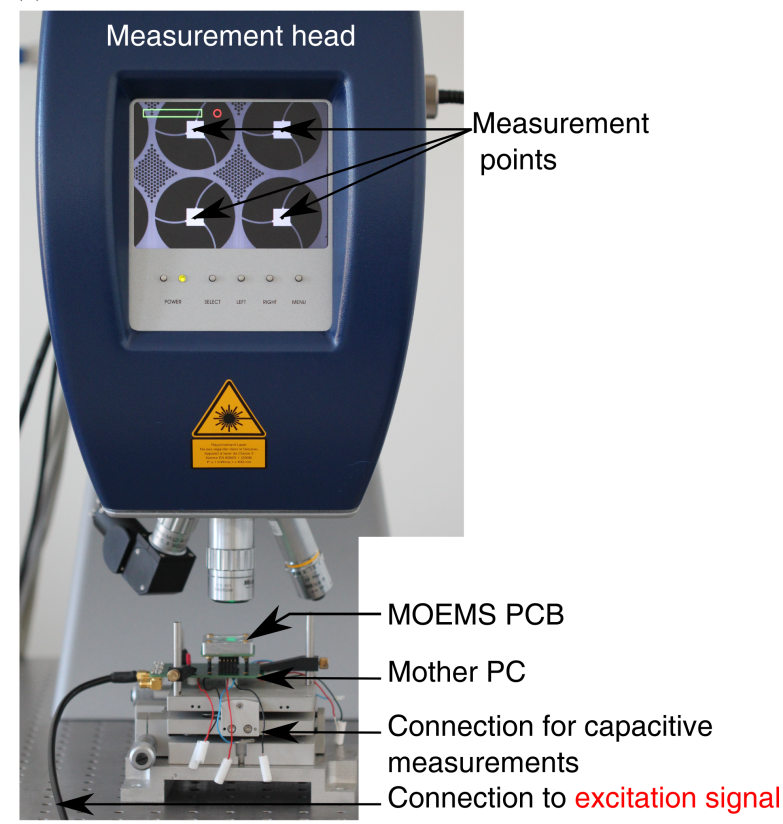

Fig. 16. MEMS analyzer setup for the displacement characterization: (a) Schematic of the connections, (b) Photograph of the setup.

which is piston mode, was measured to be $543 \mathrm{~Hz}$. The quality factor of the system was 50 based on calculations with the spectral response $(-3 \mathrm{~dB})$ and the free-oscillations characterization (exponential decrease) (respectively, Fig. 17 and 18). For such a large platform, this quality factor is relatively high compared with the $3 \times 3$ array of individually driven micromirrors presented in [6], for which the quality factor of each micromirror is 41 . The measured resonant frequency corresponds to a vertical spring stiffness of $7.2 \mathrm{~N} / \mathrm{m}$, which is coherent with the simulations, if we take into account mass $=2 \cdot 46 \cdot 10^{-6} \mathrm{~kg}$. Finally in Fig. 17, the peak corresponding to the second and third mode cannot be seen because it is too small when symmetric actuation is provided by driving of the four actuators. Then the torsional modes will not interfere when piston mode vertical actuation at the resonant frequency is performed. Static measurements were done by applying simultaneously a square signal to the 4 actuators at very low frequency $(10 \mathrm{~Hz})$ so that doppler vibrometry could be used. The static displacement was retrieved as a function of the peak-to-peak amplitude of the excitation square signal, between $0 \mathrm{~V}$ and the actuation voltage $(0-40 \mathrm{~V})$. Due to a limitation of the internal excitation signal of the MSA-500, an external amplifier was connected between the signal excitation output and the MOEMS (Fig. 16 (a)). The static displacement is plotted as a function of the applied voltage for voltages between 0 and $40 \mathrm{~V}$ in Fig. 19. The amplitude of vertical displacement was measured between 0 and approximately $2.8 \mu \mathrm{m}$, slightly depending on the gap fingers. Though, the measured static displacement curve is closer to the simulated curve obtained for $4 \mu \mathrm{m}$ gap between moving fingers. This result is consistent with the SEM measurements of the fabricated structures that show a finger gap close to $4 \mu m$.

For the phase shifting, it is important to know the displacement amplitude of all the mirrors. The dynamic displacement of the platform at resonant frequency was investigated at different points of the platform, i.e. at the position of the four micromirrors (see Fig. 2,(b)) forming a quarter of the platform (Fig. 20). The actuation voltage is set to $V_{d}=1+1 \times \sin \left(w_{0} t\right) V$. Note that the electrostatic force is proportional to the square of the voltage. Then, with the applied voltage $V_{d}$, the obtained force is a sum of a DC force, a force 

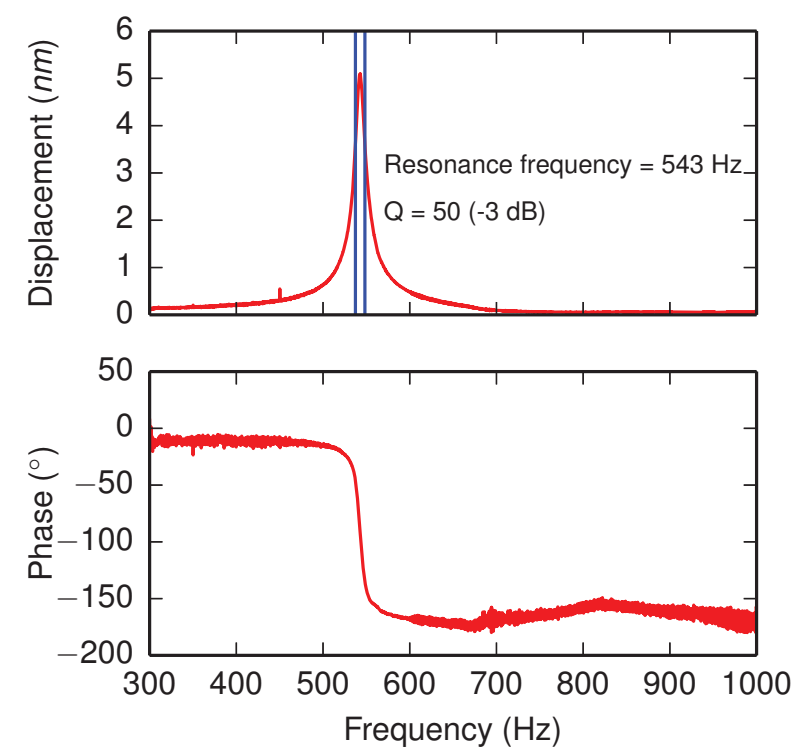

Fig. 17. Spectral response analysis of the device: (a) displacement, (b) phase.

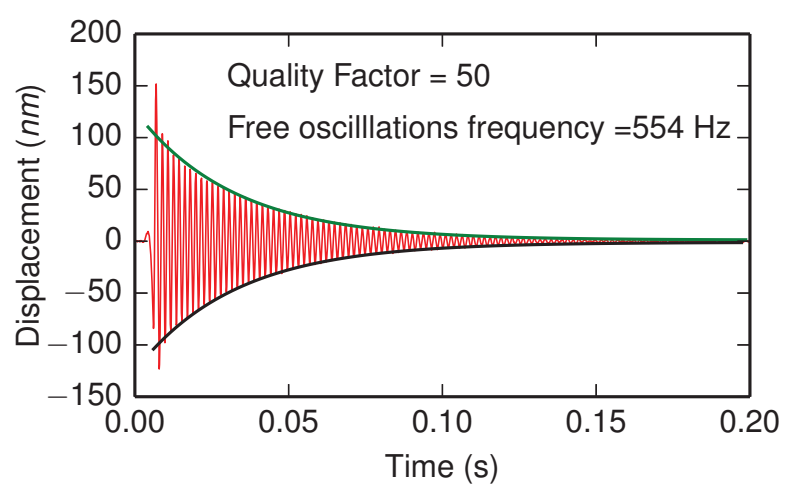

Fig. 18. Free oscillation in response to a square signal (0-5V).

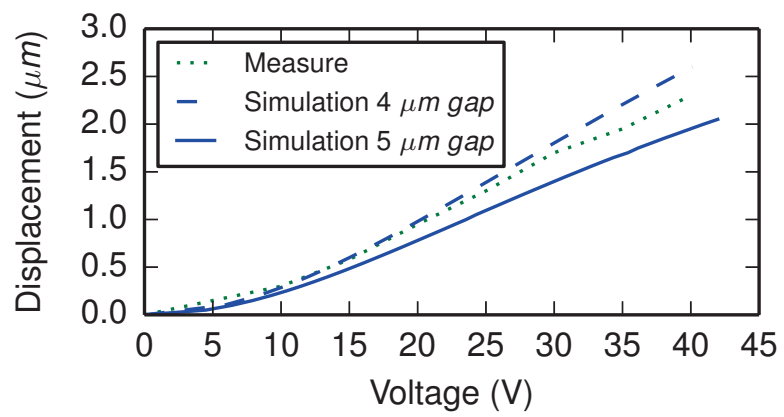

Fig. 19. Static displacement as a function of applied voltage, measured by Laser Doppler vibrometry.

proportional to $\sin \left(w_{0} t\right)$ and a force proportional to $\sin \left(2 w_{0} t+\frac{\pi}{2}\right)$. Here, the quality factor $\mathrm{Q}$ of the first mode $\left(w_{0}\right)$ is much higher so that the signal in $\sin \left(2 w_{0} t+\frac{\pi}{2}\right)$ is negligible and the dynamic displacement is synchronous with the driving voltage. Moreover, here, only dynamic displacements were measured and not 
the DC displacement. The measured displacement, much larger than the targeted one, shows the potential of this microscanner for medical imaging applications, for which small voltages are particularly important since the device is in contact with the patient. The displacement of the micromirror in the middle of the platform is $70 \mathrm{~nm}$ higher than the one next to the spring because of the vertical mechanical deformation of the suspended platform. Sasaki et al. studied the impact of the error of the displacement amplitude on the sinusoidal modulation in interferometric applications [15]. This displacement of $70 \mathrm{~nm}$ is within the tolerances presented in the article.

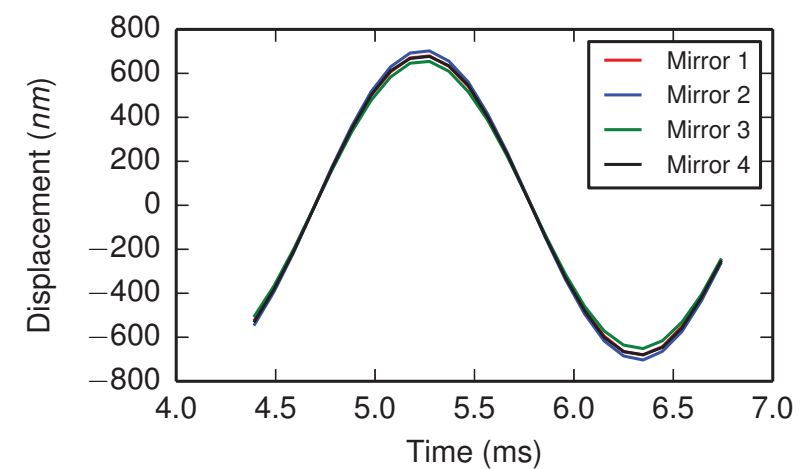

Fig. 20. Vertical displacement of the micromirror under dynamic driving voltage: (a) Mirror 1, (b) Mirror 2, (c) Mirror 3, (d) Mirror 4.

Finally, characterization of the sensing function has been performed. An impedance analyser (Agilent HP $4194 \mathrm{~A}$ ) is used to measure successively the capacitance of all the sensors as a function of applied voltage. The continuous polarization of the microactuators is obtained by an association of batteries in series $(5 \times 9 \mathrm{~V})$ to limit noise effects during the measurements. A high nominal capacitance was measured around $50 \mathrm{pF}$ with a precision of $\pm 5 f F$. This can be explained by parasitic capacitances. However, here, the variations of the capacitances are interesting to retrieve the vertical displacement. Therefore this nominal capacitance, i.e. the capacitance measured at $V_{\text {actuation }}=0 \mathrm{~V}$, which is stable during the measurement time, has been subtracted from the measured values. The measures were taken at $f=1.1 \mathrm{MHz}$, and eight measures were used to obtain each average value. The oscillation level of the impedance analyser was set to $0.5 \mathrm{~V}$. The capacitances were measured as a function of the applied voltage and then retrieved as a function of the vertical displacement using the results presented in Fig. 19. The differential capacitance of each sensor, i.e. the capacitance of Configuration 1 minus the capacitance of Configuration 2, is plotted as a function of the vertical displacement in Fig. 21. The sensitivities for each sensors correspond to the simulated sensitivity for a gap of $4 \mu \mathrm{m}$, i.e. $27 \mathrm{fF} / \mu \mathrm{m}$. One of the four sensors has a higher sensitivity of $36 \mathrm{fF} / \mu \mathrm{m}$. One explanation is that the platform is slightly tilted toward the side corresponding to this sensor creating an offset. Also a local structural defect can explain such a small increase of the sensitivity. 
(a)

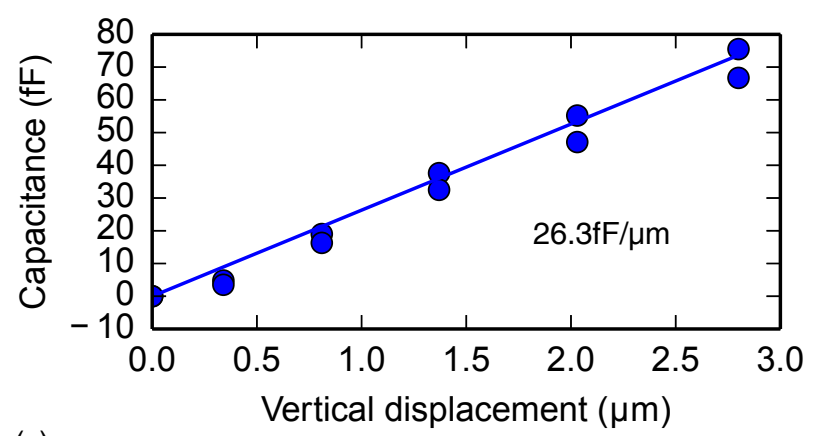

(c)

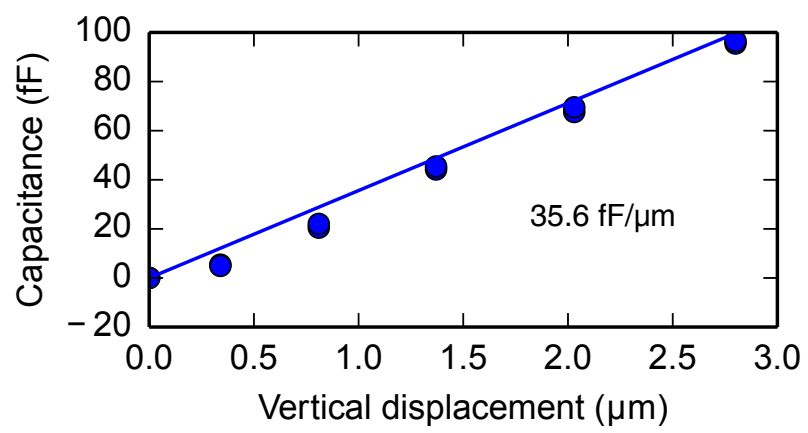

(b)

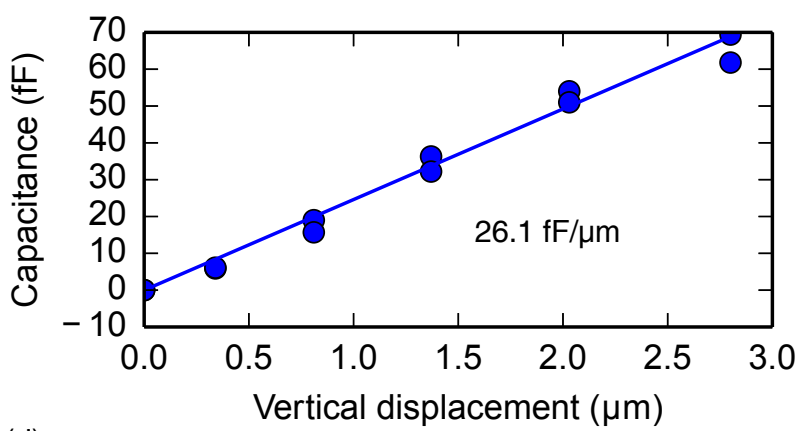

(d)

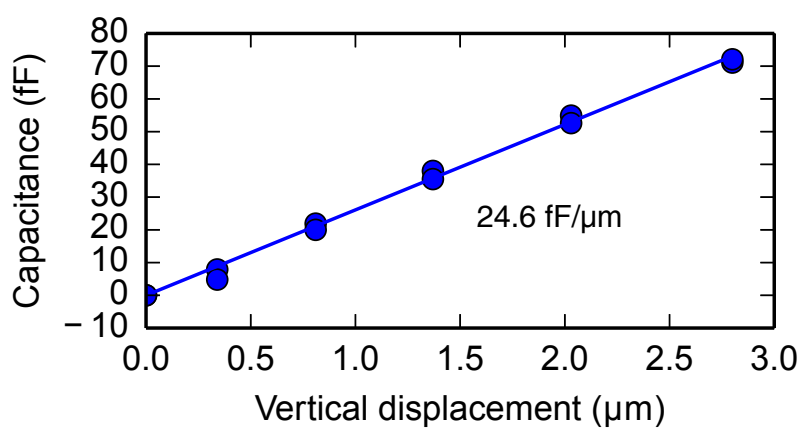

Fig. 21. Measured differential capacitance for each sensor of a chip and their corresponding sensitivity: (a) Sensor 1, (b) Sensor 2, (c) Sensor 3, (d) Sensor 4.

\section{Conclusion}

We report the design, the simulation and the fabrication of a micromirror electrostatic vertical microscanner which is a key component of a multi-channel "active" Mirau microinterferometer. Each functional part of the microscanner is developed according to the optical design of the microinterferometer in which the microscanner is to be integrated. In particular, the fabrication process is optimized and described in details for typical critical steps in MOEMS fabrication, e.g. protection of optical coatings, asymmetric vertical comb-drive etching, release of an actuated structure and chip separation. Finally, the characterization confirms that the microscanner meets the optical and mechanical targeted specifications. For the implementation of the phase modulating algorithm, the microscanner is driven sinusoidally at its resonance frequency $(\sim 500 \mathrm{~Hz})$. The use of such technique leads to a enhancement of the dynamic signal of the optical system. This miniaturized multi-channel interferometer shows the potential of MOEMS for the development of vertically integrated microsystems for applications in biomedical imaging. Future perspectives include the vertical wafer-level assembly of the microlens wafer, the micromirror wafer and the beam splitter wafer for the realization of the "active" Mirau microinterferometer. This vertical assembly is based on a silicon-glass anodic bonding. This process is potentially dangerous for the final optical quality of the micromirrors due to the high bonding temperature and for the microactuator due to the generated electrical field. Hence these steps require further work. Finally, the whole stack must be diced into individual chips which is not trivial due to the total thickness of the stack $(5 \mathrm{~mm})$.

\section{Acknowledgement}

The authors thank Gilles Bourbon for his insights and for fruitful scientific discussions. This work is financed by the collaborative project VIAMOS of the European Commission (FP7, ICT program, grant no. 
318542) and supported by the Labex Action program (ANR-11-LABX-0001-01), by the French RENATECH network, by the Collegium SMYLE and by the FEMTO-ST institute facilities.

\section{References}

1. James G Fujimoto. Optical coherence tomography for ultrahigh resolution in vivo imaging. Nat. Biotechnol., 21(11):1361-7, November 2003.

2. Dae Yu Kim, John S Werner, and Robert J Zawadzki. Comparison of phase-shifting techniques for in vivo full-range, high-speed Fourier-domain optical coherence tomography. J. Biomed. Opt., 15(October 2010):056011, 2010.

3. Li Fan, Wibool Piyawattanametha, Ming C. Wu, Aaron D. Aguirre, Paul R. Herz, Yu Chen, and James G. Fujimoto. High-resolution 3D OCT imaging with a MEMS scanning endoscope. In MOEMSMEMS Micro \& Nanofabrication, volume 5719, pages 140-143, January 2005.

4. Abraham P Lee, Charles F. McConaghy, Gary Sommargren, Peter Krulevitch, and Eugene W Campbell. Vertical-actuated electrostatic comb drive with in situ capacitive position correction for application in phase shifting diffraction interferometry. J. Microelectromechanical Syst., 12(6):960-971, 2003.

5. Hyuck Choo, Rishi Kant, and David Garmire. Fast, MEMS-based, phase-shifting interferometer. In Solid-State Sens. Actuator Work., pages 94-95, 2006.

6. Jin-Chern Chiou, Chen-Chun Hung, Li-Jung Shieh, and Zhao-Long Tsai. Novel electrostatic MOEMS phase shifter array using CMOS-MEMS process. J. Micro/Nanolithography, MEMS MOEMS, 9:013030, 2010 .

7. Kentaro Oda, Hidekuni Takao, Kyohei Terao, Takaaki Suzuki, Fusao Shimokawa, Ichirou Ishimaru, and Fumikazu Oohira. Vertical comb-drive MEMS mirror with sensing function for phase-shift device. Sensors Actuators, A Phys., 181:61-69, 2012.

8. O G Karhade, F L Degertekin, and T R Kurfess. SOI-based micro scanning grating interferometers: device characterization, control and demonstration of parallel operation. J. Micromech. Microeng., 18:045007, 2008.

9. Malgorzata Kujawinska, Kay Gastinger, Michal Jozwik, Karl Henrik Haugholt, Christoph Schaeffel, and Stephan Beer. An interferometric test station for massive parallel inspection of MEMS and MOEMS. Photonics Lett. Pol., 1(2):58-60, 2009.

10. J Albero, S Bargiel, N Passilly, P Dannberg, M Stumpf, U D Zeitner, C Rousselot, K Gastinger, and C Gorecki. Micromachined array-type Mirau interferometer for parallel inspection of MEMS. J. Micromech. Microeng., 21(6):065005, June 2011.

11. Wei Xu, Alain Bosseboeuf, Fabien Parrain, and Emile Martincic. Design of a long range bidirectional MEMS scanner for a tunable 3D integrated Mirau interferometer. In Symp. Des. Test, Integr. Packag. MEMS/MOEMS, pages 1-6. IEEE, April 2014.

12. Else Gallagher, Walied Moussa, and Mark McDermott. A review of fabrication processes for vertical comb drives. Microsyst. Technol., 18(4):381-397, January 2012.

13. J. Krauter, T. Boettcher, W. Lyda, W. Osten, N. Passilly, L. Froehly, S. Bargiel, J. Albero, S. Perrin, J. Lullin, and C. Gorecki. Optical design of a vertically integrated array-type Mirau-based OCT system. In Photonics Eur., page 91320L, 2014.

14. Peng Bu, Xiangzhao Wang, and Osami Sasaki. Full-range parallel Fourier-domain optical coherence tomography using sinusoidal phase-modulating interferometry. J. Opt. A Pure Appl. Opt., 9:422-426, 2007. 
15. O Sasaki and H Okazaki. Analysis of measurement accuracy in sinusoidal phase modulating interferometry. Appl. Opt., 25(18):3152, 1986.

16. Wei-Shan Wang, Justine Lullin, Joerg Froemel, Maik Wiemer, Sylwester Bargiel, Nicolas Passilly, Christophe Gorecki, and Thomas Gressner. Multi-wafer bonding technology for the integration of a micromachined Mirau interferometer. In Proc. SPIE, volume 1, pages 3-9, 2015.

17. Jorge Albero, Stéphane Perrin, Sylwester Bargiel, Nicolas Passilly, Maciej Baranski, Ludovic GauthierManuel, Florent Bernard, Justine Lullin, Luc Froehly, Johann Krauter, Wolfgang Osten, and Christophe Gorecki. Dense arrays of millimeter-sized glass lenses fabricated at wafer-level. Opt. Express, 23(9):11702, 2015.

18. J E Harvey and C Ftaclas. Diffraction effects of telescope secondary mirror spiders on various imagequality criteria. Appl. Opt., 34(28):6337-6349, October 1995.

19. J L Richter. Spider diffraction: a comparison of curved and straight legs. Appl. Opt., 23(12):1907-1913, June 1984.

20. Justine Lullin, Sylwester Bargiel, Emilie Courjon, Stephane Perrin, Maciej Baranski, Nicolas Passilly, and Christophe Gorecki. Monolithic integration of a glass membrane on silicon micro-actuator for micro-interferometry. In 2014 Int. Conf. Opt. MEMS Nanophotonics, pages 87-88. IEEE, August 2014.

21. Justine Lullin, Stephane Perrin, Maciej Baranski, Sylwester Bargiel, Luc Froehly, Nicolas Passilly, Jorge Albero, and Christophe Gorecki. Impact of mirror spider legs on imaging quality in Mirau microinterferometry. Opt. Lett., 40(10):2209-2212, May 2015.

22. Kieth Fedder. Simulation of Microelectromechanical Systems. PhD thesis, 1994.

23. Toshiyuki Tsuchiya and Hirofumi Funabashi. A z-axis differential capacitive SOI accelerometer with vertical comb electrodes. Sensors Actuators A Phys., 116(3):378-383, October 2004.

24. Jiankun Wang, Zhenchuan Yang, and Guizhen Yan. Silicon-on-insulator out-of-plane electrostatic actuator with in situ capacitive position sensing. J. Micro/Nanolithography, MEMS, MOEMS, 11(3):033006, July 2012. 